\title{
Results of optical monitoring of 5 SDSS double QSOs with the Nordic Optical Telescope
}

\author{
D. Paraficz ${ }^{1}$, J. Hjorth ${ }^{1}$, and Á. Elíasdóttir ${ }^{1,2}$ \\ 1 Dark Cosmology Centre, Niels Bohr Institute, University of Copenhagen, Juliane Maries Vej 30, 2100 Copenhagen, Denmark \\ e-mail: danutas@dark-cosmology.dk \\ 2 Department of Astrophysical Sciences, Princeton University, Princeton, NJ08544, USA
}

Received 20 November 2008 / Accepted 25 February 2009

\begin{abstract}
We present optical $R$-band light curves of five SDSS double QSOs (SDSS J0903+5028, SDSS J1001+5027, SDSS J1206+4332, SDSS J1353+1138, SDSS J1335+0118) obtained from monitoring at the Nordic Optical Telescope (NOT) between September 2005 and September 2007. We also present analytical and pixelated modeling of the observed systems. For SDSS J1206+4332, we measured the time delay to be $\Delta \tau=116_{-5}^{+4}$ days, which, for a singular isothermal ellipsoid model, corresponds to a Hubble constant of $73_{-4}^{+3} \mathrm{~km} \mathrm{~s}^{-1} \mathrm{Mpc}^{-1}$. Simultaneous pixelated modeling of five other systems for which a time delay has now been previously measured at the NOT leads to $H_{0}=61.5_{-4}^{+8} \mathrm{~km} \mathrm{~s}^{-1} \mathrm{Mpc}^{-1}$. Finally, by comparing light curves of the two images of each system, suitably shifted by the predicted or observed time-delays, we found no evidence of microlensing variability over the course of the monitoring period.
\end{abstract}

Key words. gravitational lensing - cosmological parameters - quasars: general

\section{Introduction}

A gravitationally lensed quasar is a quasar (QSO) that is lensed by a massive foreground object such as a galaxy or a group of galaxies. If the lens is close enough to the line of sight then the quasar is strongly lensed and will be multiply imaged. Due to the different travel times for each light path, any intrinsic variation in the quasar is observed in the images at different times. This time difference, referred to as the time delay, can be measured by comparing the light curves of the images. As first shown by Refsdal (1964), the Hubble constant can be determined from the time delay provided that the mass distribution is known. Conversely, for a fixed Hubble constant, the mass distribution of the lensing galaxy can be constrained.

Determining the Hubble constant from the time delays between light variations in multiple images of gravitationally lensed QSOs is a classical application of lensing in cosmology, but despite 25 years of effort, results remain inconclusive. This partly stems from incomplete knowledge of the mass distribution along the light path to the QSO and partly from the perturbing effects of microlensing on sparsely sampled light curves of the underlying long-term variations.

To address these issues, we have conducted a monitoring program at the Nordic Optical Telescope (NOT) with the aim of obtaining densely sampled light curves of five lensing systems (SDSS J0903+5028, SDSS J1001+5027, SDSS J1206+4332, SDSS J1353+1138, SDSS J1335+0118). Dense sampling is required to quantify the effects of microlensing (Paczyński 1986; Schild 1996; Paraficz et al. 2006) and to maximize chances of determining a time delay from a relatively short monitoring campaign.
Estimating the Hubble constant using time delay measurements is strongly dependent on the underlying mass distribution and hence the choice of lens model (Oguri 2007). Two different approaches to modeling lenses are commonly used. The first one, the non-parametric method (Saha \& Williams 1997), generates many models that perfectly fit the data, each of them giving a different time delay which can be then averaged. For the second method, the analytical method (Keeton 2001), one assumes physical properties of the mass distribution of the lens. Comparison of the two approaches gives a useful indication of the systematic errors in e.g. the Hubble constant determined this way.

Luckily, it seems that simple lens models are very good first approximations of the real mass distributions of lenses (Koopmans et al. 2006). Therefore we chose to use the singular isothermal potential to analytically model all the lenses we observe.

In this paper we present the results of a monitoring campaign at the NOT of 5 doubly lensed quasars. We measured the time delay of one of the lensing systems, SDSS J1206+4332, by analyzing light curves of the two quasar images obtained from six months of monitoring, thereby demonstrating the feasibility of short-term monitoring for time-delay measurement. Based on our measurement of the time delay and 5 other time-delay measurements previously obtained at the NOT, we estimated the Hubble constant.

Section 2 describes the details of our monitoring campaign and Sect. 3 introduces the observed targets. The photometric technique based on image deconvolution is described in Sect. 4 and the light curves of all 5 targets are presented in Sect. 5. In Sect. 6 the time delay of SDSS J1206+4332 is determined. In 
Sect. 7 we perform a microlensing search in the 5 quasar light curves and estimate upper limits to the microlensing signal. In Sect. 8 we perform analytical and pixeleted modeling. Section 9 is devoted to simultaneous modeling of 5 NOT-determined time delay systems with the aim of a joint Hubble constant estimate. In Sect. 10 we discuss the results.

In the paper we use a flat $\Lambda \mathrm{CDM}$ Universe, $\Omega_{\mathrm{m}}=0.3$ and $\Omega_{\Lambda}=0.7$.

\section{Observation}

The observations were gathered from monitoring programs carried out in the periods September-March 2005/2006, October-March 2006/2007 and April-September 2007 at the Nordic Optical Telescope (NOT); a 2.5-m telescope located at Roque de los Muchachos in La Palma, Spain. The advantage of this telescope is its fairly flexible scheduling, which made almost nightly monitoring possible. Our targets were observed every night under all three operative modes at the NOT: observer, service and technical. However there were severe obstacles preventing frequent sampling: bad weather, Guaranteed Time programs at the NOT and sensitivity of the deconvolution software to imperfectness of the data.

The detectors used in the monitoring were chosen in order to obtain the most frequent sampling and the best image quality. Thus, we used ALFOSC (The Andalucia Faint Object Spectrograph and Camera; pixel scale 0.'189) whenever it was mounted on the telescope and StanCam (the Stand-by CCD Camera, which is permanently mounted at the NOT; pixel scale $0 . ' 176$ ), otherwise. The seeing varied from $0 .{ }^{\prime} 4$ to 3 '. $^{\prime \prime} 0$ with 1 .' 0 being the most frequent value.

We observed the objects in the $R$ band, only. The pilot phase of the program involved monitoring of a gravitationally lensed system SDSS J0903+5028 at $z=3.6$ (Johnston et al. 2003) for 10 min every night. This system contains two quasar images (see Table 1) separated by 2 .' 8 and aligned on opposite sides of the lensing galaxy at the redshift $z=0.388$ (Johnston et al. 2003).

After the first observing season we decided to observe three lensed systems alternately, SDSS J1001+5027 and SDSS J1206+4332 together in one night for $5 \mathrm{~min}$ each, and SDSS J0903+5028 every second night for $10 \mathrm{~min}$. The third observing season was again divided in two groups. We continued observing SDSS J1001+5027 and SDSS J1206+4332 for 5 min each every second night, alternately with two new targets, SDSS $\mathrm{J} 1335+0118$ (5 min) and SDSS J1353+1138 (3 min). The exposure times were chosen so that the signal-to-noise ratio of the fainter component of each system would be above 10. Finding charts of all the targets are presented in Figs. 1 and 2.

One of the major challenges in monitoring gravitationally lensed quasars is the lack of prior knowledge of time delay. Thus, time-delay measurements require well-sampled light curves with accurate photometry over a period of time substantially longer than the time delay. Because the time delay is not known, one has to calculate a theoretical time delay before planning the observations.

Assuming that the theoretical prediction of the time delay for a given system is a good approximation (which is not always the case) there is still a question as to whether the quasar will vary during the period of monitoring and what will be the timescale and amplitude of the variations. Quasar brightness might vary from a day up to years independently of the mass of the black hole (Wold et al. 2007). We know that the rapid variability implies a light source at very small distance from the black hole (Webb \& Malkan 2000), while variations on long time-scales

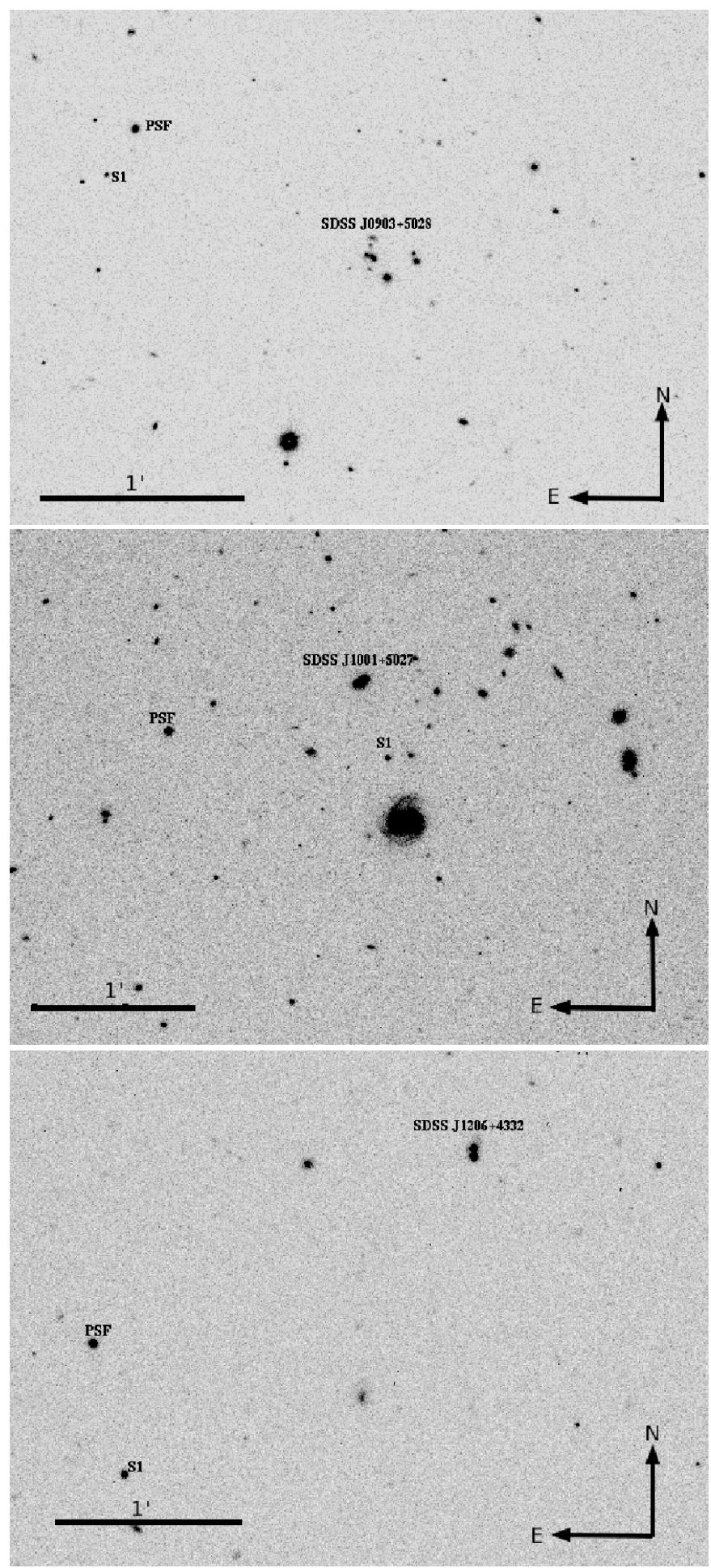

Fig. 1. Finding chart of the three systems: top: SDSS J0903+5028, middle: SDSS J1001+5027, bottom: SDSS J1206+4332. The reference star (S1) and the star used to model the PSF are indicated.

are related to morphological changes of jets on parsec scales or to accretion-disk instabilities (de Vries et al. 2006). However, it is impossible to predict whether a quasar will vary or not within a given period and timescale, which is why the success of each quasar monitoring is uncertain.

Another difficulty with gravitationally lensed quasar observations is microlensing by stars in the lensing galaxy. 


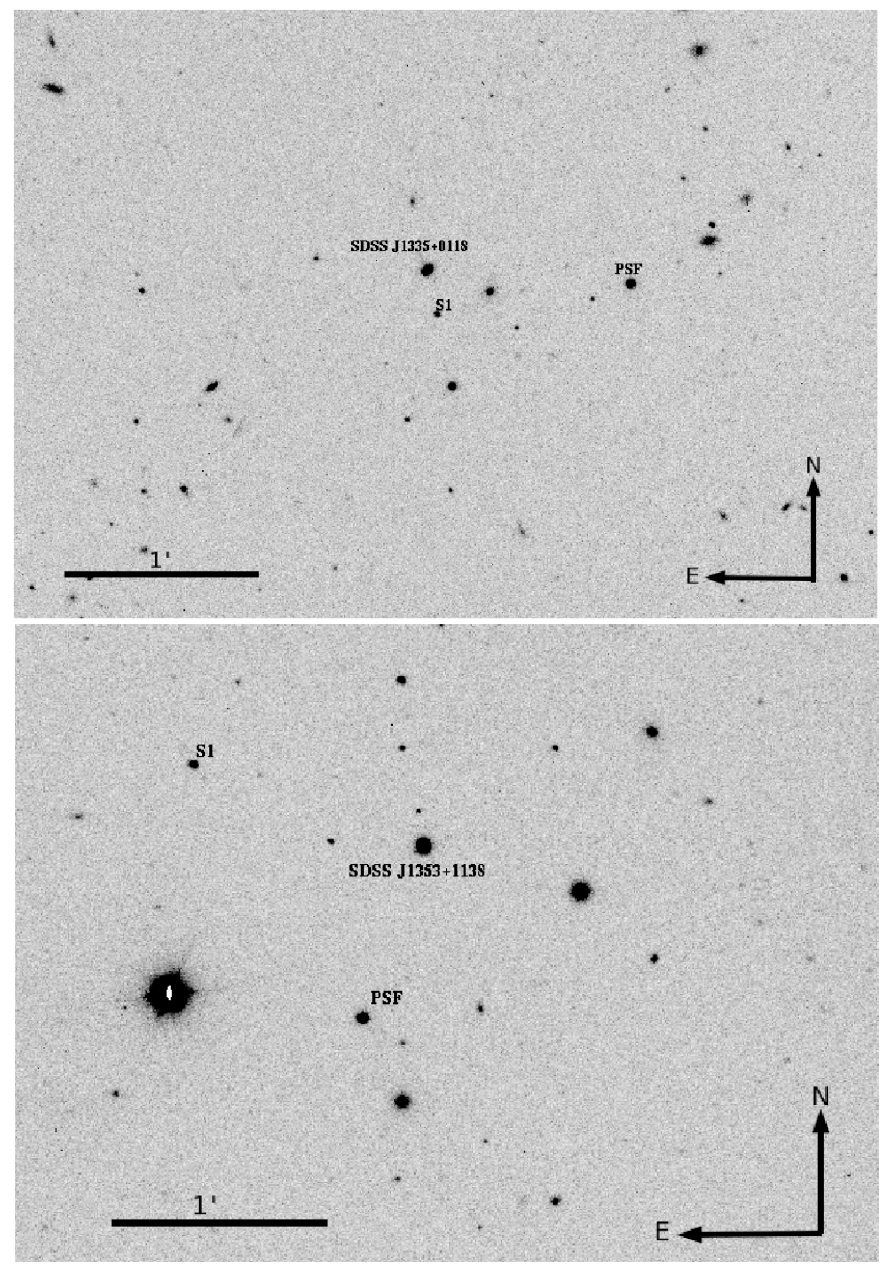

Fig. 2. Finding chart of the two systems. Top: SDSS J1335+0118 and bottom: SDSS J1353+1138. The reference star and the star used to model the PSF are indicated.

Microlensing can change the results by about $0.5-1.0 \mathrm{mag}$ and it is completely unpredictable (Chang \& Refsdal 1979).

Thus, in order to get a time delay one has to monitor quasars with high sampling, so that events like microlensing can be extracted from the quasar variability in further analysis. Our monitoring program was designed to minimize this problem.

Observation planning, monitoring supervision and image reduction was made by the first author during her stay at the NOT. Data reduction was performed using IRAF reduction utilities.

\section{Targets}

The individual targets observed are introduced below. The astrometry and redshifts of the quasar images and the lenses are summarized in Table 1.

\subsection{SDSS J0903+5028}

SDSS J0903+5028, a doubly lensed quasar system, was discovered from the Sloan Digital Sky Survey by Johnston et al. (2003). Using the ARC $3.5 \mathrm{~m}$ telescope it was found that the system has two quasar images separated by 2 ". 8 with the lens, a red galaxy $(z=0.388)$, in between. Spectroscopic follow up observation at the Keck II telescope proved that the two objects are the
Table 1. Astrometric properties and redshifts ${ }^{1}$.

\begin{tabular}{|c|c|c|c|}
\hline & SDSS J0903+5028 & & \\
\hline Object & $\begin{array}{c}\text { RA (J2000.0) } \\
\text { (h:m:s) }\end{array}$ & $\begin{array}{l}\operatorname{Dec}(\mathrm{J} 2000.0) \\
\quad(\mathrm{d}: \mathrm{m}: \mathrm{s})\end{array}$ & $z$ \\
\hline $\mathrm{A}$ & 090335.13 & +502820.21 & 3.6 \\
\hline B & 090334.88 & +502818.75 & 3.6 \\
\hline G & 090334.93 & +502819.53 & 0.388 \\
\hline & SDSS J1001+5027 & & \\
\hline Object & $\begin{array}{l}\text { RA (J2000.0) } \\
\quad(\mathrm{h}: \mathrm{m}: \mathrm{s})\end{array}$ & $\begin{array}{c}\operatorname{Dec}(\mathrm{J} 2000.0) \\
(\mathrm{d}: \mathrm{m}: \mathrm{s})\end{array}$ & $z$ \\
\hline$\overline{\mathrm{A}}$ & 100128.61 & +502756.9 & 1.838 \\
\hline \multirow[t]{2}{*}{ B } & 100128.35 & +502758.5 & 1.838 \\
\hline & $\begin{array}{c}x \\
(\operatorname{arcsec})\end{array}$ & $\begin{array}{c}y \\
(\operatorname{arcsec})\end{array}$ & $z$ \\
\hline G1 & $1.779 \pm 0.049$ & $0.857 \pm 0.123$ & $0.2 \leq z \leq 0.5$ \\
\hline $\mathrm{G} 2$ & $1.795 \pm 0.088$ & $-0.700 \pm 0.053$ & $0.2 \leq z \leq 0.5$ \\
\hline Object & $\begin{array}{c}\text { SDSS J1206+4332 } \\
\text { RA (J2000.0) } \\
\text { (h:m:s) }\end{array}$ & $\begin{array}{c}\operatorname{Dec}(\mathrm{J} 2000.0) \\
(\mathrm{d}: \mathrm{m}: \mathrm{s})\end{array}$ & $z$ \\
\hline $\bar{A}$ & 120629.65 & +433217.6 & 1.789 \\
\hline \multirow[t]{2}{*}{ B } & 120629.65 & +433220.6 & 1.789 \\
\hline & $\begin{array}{c}x \\
(\operatorname{arcsec})\end{array}$ & $\begin{array}{c}y \\
(\operatorname{arcsec})\end{array}$ & $z$ \\
\hline G1 & $-0.664 \pm 0.137$ & $1.748 \pm 0.028$ & 0.748 \\
\hline G2 & $1.320 \pm 0.147$ & $5.999 \pm 0.148$ & $\geq 0.7$ \\
\hline \multirow[t]{3}{*}{ G3 } & $+2.052 \pm 0.200$ & $2.397 \pm 0.152$ & blue \\
\hline & SDSS J1335+0118 & & \\
\hline & $\begin{array}{c}x \\
(\operatorname{arcsec})\end{array}$ & $\begin{array}{c}y \\
(\operatorname{arcsec})\end{array}$ & $z$ \\
\hline $\bar{A}$ & $0.000 \pm 0.001$ & $0.000 \pm 0.001$ & 1.57 \\
\hline B & $-1.038 \pm 0.002$ & $-1.165 \pm 0.002$ & 1.57 \\
\hline \multirow[t]{3}{*}{ G } & $-0.769 \pm 0.011$ & $-0.757 \pm 0.011$ & 0.44 \\
\hline & SDSS J1353+1138 & & \\
\hline & $\begin{array}{l}\text { RA (J2000.0) } \\
\text { (h:m:s) }\end{array}$ & $\begin{array}{c}\operatorname{Dec}(\mathrm{J} 2000.0) \\
(\mathrm{d}: \mathrm{m}: \mathrm{s})\end{array}$ & $z$ \\
\hline A & 135306.35 & +113804.81 & 1.63 \\
\hline B & 135306.08 & +113801.43 & 1.63 \\
\hline G & 133506.10 & +113800.39 & $\sim 0.3$ \\
\hline
\end{tabular}

${ }^{1}$ All $x, y$ positions are relative to the A component and their errors do not include the error of the image scale. The positive direction of the those coordinates are to the west and north, respectively.

images of one quasar at $z=3$.6. Johnston et al. (2003) concluded that other galaxies in the vicinity of the lensing galaxy might be gravitationally bound with the lens, adding external shear to the lensed system.

\subsection{SDSS J1001+5027 and SDSS J1206+4332}

SDSS J1001+5027 and SDSS J1206+4332 were recognized as lensing candidates in a strong lensing survey using the Sloan Digital Sky Survey. Oguri et al. (2005) photometrically and spectroscopically confirmed using the ARC $3.5 \mathrm{~m}$ and University of Hawaii 2.2-m telescopes, that those two targets are indeed lensing systems. They reported that SDSS J1001+5027 and SDSS J1206+4332 have image separation of 2'. 86 and 2'.90 respectively with the source objects being quasars at $z=1.838$ and $z=1.789$.

Oguri et al. (2005) noted that the two lensing galaxies of SDSS J1001+5027 have colors consistent with those of earlytype galaxies at $0.2 \leq z \leq 0.5$. SDSS J1206+4332 appears to have three lensing galaxies where the main lens, G1, has an associated absorber at redshift $z=0.748$, the second galaxy, $\mathrm{G} 2$, is identifed as a high redshift galaxy $z \leq 0.7$ and, the third galaxy, 
G3, is a blue galaxy. Oguri et al. (2005) using the lensmodel software (Keeton 2001) modeled the two systems showing that they are both strongly affected by the potential of more than one galaxy.

\subsection{SDSS $J 1335+0118$}

SDSS J1353+1138 is a doubly lensed system, discovered in the Sloan Digital Survey by Oguri et al. (2004). The photometric follow up made at the Subaru 8.2-m and Keck I telescopes confirmed that the system consists of two gravitationally lensed images separated by 1".56 with a single lensing galaxy in the centre. Spectroscopic observations made at the ESO New Technology Telescope (NTT) showed that the A and B components of the system are images of a quasar at redshift $z=1.57$. Eigenbrod et al. (2006b) reported that the lensing galaxy is a low-redshift galaxy with $z=0.44$.

\subsection{SDSS $J 1353+1138$}

Inada et al. (2006) discovered the SDSS J1353+1138 lensed quasar system from SDSS lensed quasar survey. For imaging and spectroscopy Inada et al. (2006) used the University of Hawaii 2.2-m telescope, the Keck I and II telescopes and the Magellan Consortium's Landon Clay 6.5-m telescope. The observations showed that the two quasar components separated by 1". 41 have redshift $z=1.629$ and the lensing galaxy in between the images is at $z \sim 0.3$.

\section{Photomery and image deconvolution}

\subsection{Image deconvolution}

Time delay estimation requires high precision photometry separately for each lensing image. Since our targets are small angular separation systems we need to use a mathematical method, deconvolution, to separate the images.

An observed image is a convolution of a real light distribution with the so-called total blurring function or point spread function (PSF). The goal of the deconvolution is to extract the brightness of a source using knowledge about the PSF. Deconvolution is an inverse problem without a unique solution. The method used for choosing the best solution is minimization of the difference between a model and a real image.

All data used in this paper were deconvolved using the PSF Controled Deconvolution software created by Magain et al. (1998). In this software the input are the position and intensities of the images, which need to be well defined to succeed. We take the image positions from previous studies because these were better resolved and the quasar postions well determined (see Table 1).

In Fig. 3 we present the deconvolution results for the observed targets. On the left are shown the deconvolved images (mathematical models of lensing systems) and on the right the data images. We can see that the deconvolution reveals not only the quasar images but also the lensing galaxies which are not visible in the raw data.

\subsection{Photometry}

Photometry of all objects presented in this paper was made using the PSF Controled Deconvolution software (Magain et al. 1998). The software deconvolves all frames of a given object

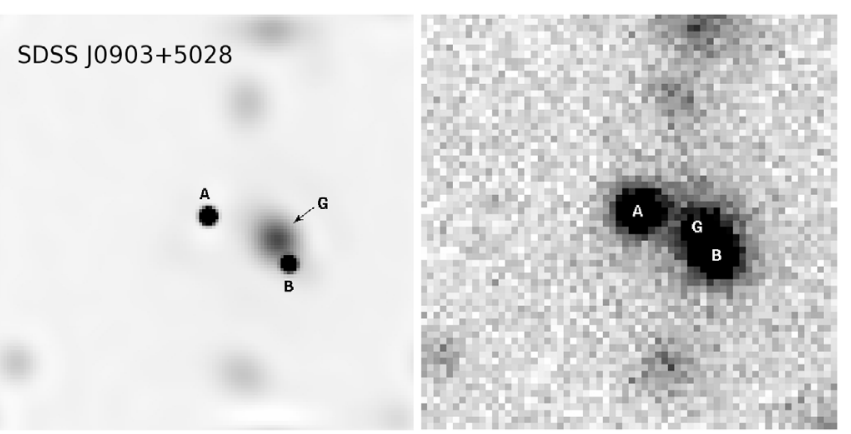

SDSS J1001+5027
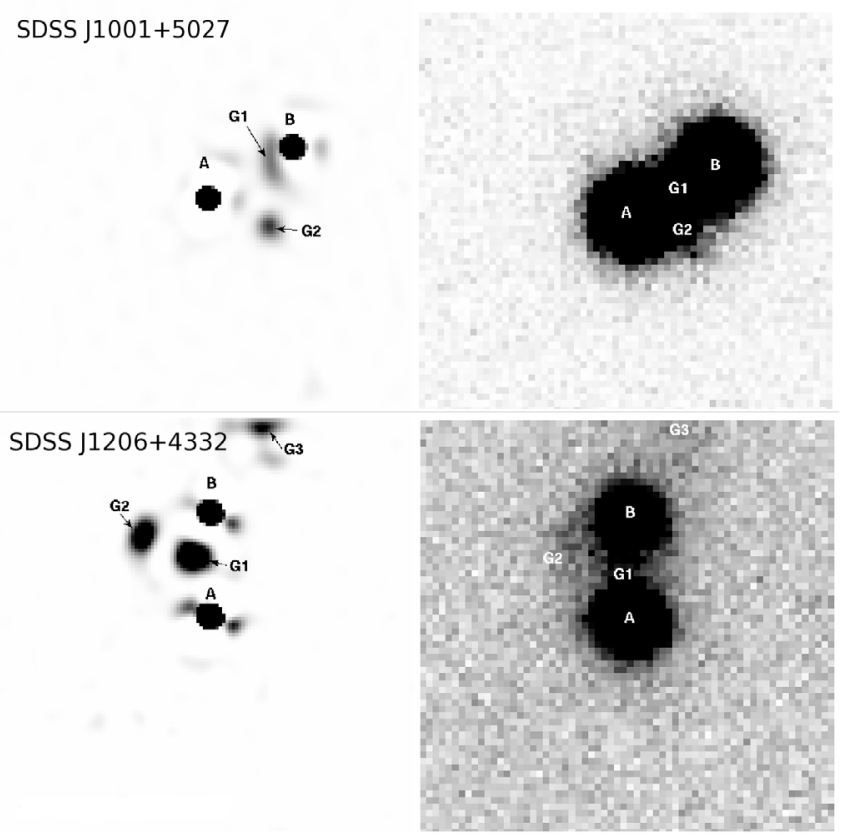

SDSS J1335+0118
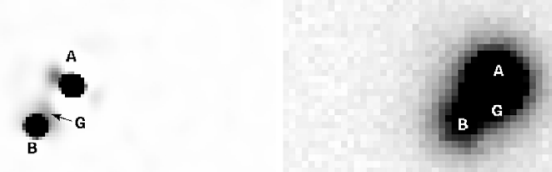

SDSS $1353+1138$
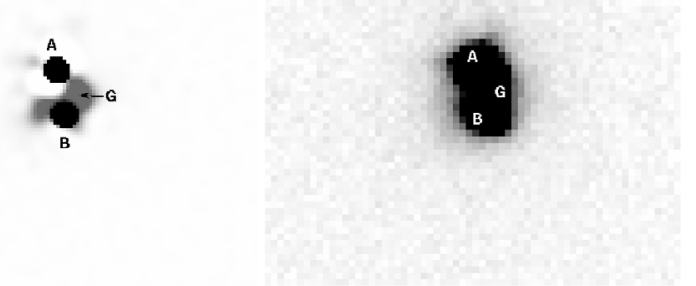

Fig. 3. Results of the deconvolution of the 5 systems. On the left are deconvolved images and on the right original images. The deconvolution reveals not only quasar images but also the lensing galaxy which is otherwise not visible.

simultaneously, it constrains the position of the images and lensing galaxies and allows the magnitude of the quasar images 


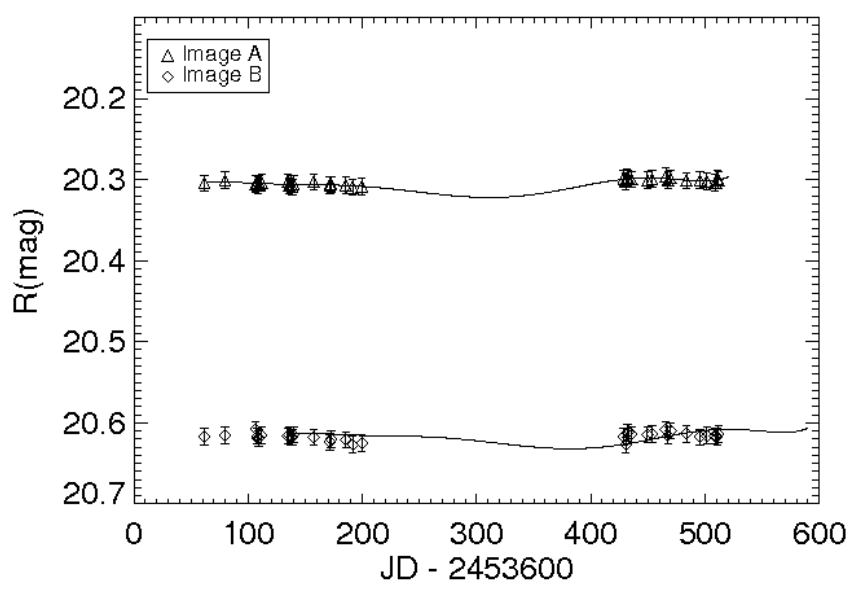

Fig. 4. $R$-band light curves of SDSS J0903+5028. A polynomial is fitted to the light curve of A counterpart. This polynomial was also fitted to the B image with magnitude and time shift (see Table 2).

to vary freely. The algorithm has been implemented in various analysis of quasars (Burud et al. 2000, 2002a,b; Hjorth et al. 2002; Jakobsson et al. 2005; Vuissoz et al. 2007; Eigenbrod et al. 2006a).

The light curves of SDSS J0903+5028, SDSS J1335+0118, SDSS J1353+1138, SDSS J1001+5027, SDSS J1206+4332 consist of $34,31,31,24,34 R$-band data points, respectively, as presented in Figs. 4-8. Data were taken from two optical cameras, ALFOSC (marked in black) and StanCam (marked in blue). The magnitudes are calculated relative to the PSF magnitude marked on the finding charts (Figs. 1 and 2). Due to the small field of view and comparatively small sensitivity of StanCam and lack of bright stars next to our targets the reference stars presented in the plots are only from ALFOSC. The reference star images were also deconvolved to look for systematic errors in the deconvolution and to estimate the photometric uncertainties. Assuming that reference stars are not intrinsically variable any variability seen in their light curves must be due to photometric and deconvolution uncertainties. The error bars of the quasar images and the reference stars coming from ALFOSC are the averaged photon noise and uncertainties measured from the variations of the reference stars combined in quadrature. For data points from StanCam we have assumed one standard error bar of 0.02 mag which should approximately include all uncertainties. In the case of ALOSC data of SDSS J0903+5028 and StanCam data of SDSS J1335+0118 there were no non-variable star in the field except PSF star, thus the error bars of the quasar magnitude are set to $0.02 \mathrm{mag}$, which is the approximate photometric uncertainty of the B image in average atmospheric conditions.

\section{Light curves}

\subsection{QSOs with little variability}

In 3 of the 5 systems (SDSS J0903+5028, SDSS J1335+0118, SDSS J1353+1138) no significant variability was observed. In Figs. 4-6 we plot the light curves of the three systems. By visual examination we see no variability in the quasar images.

In order to see whether small fluctuations in the light curves say something about the time delay we fitted polynomials to the light curves of the A counterpart. These were then fitted to the B light curves with magnitude and time shift. Since there are

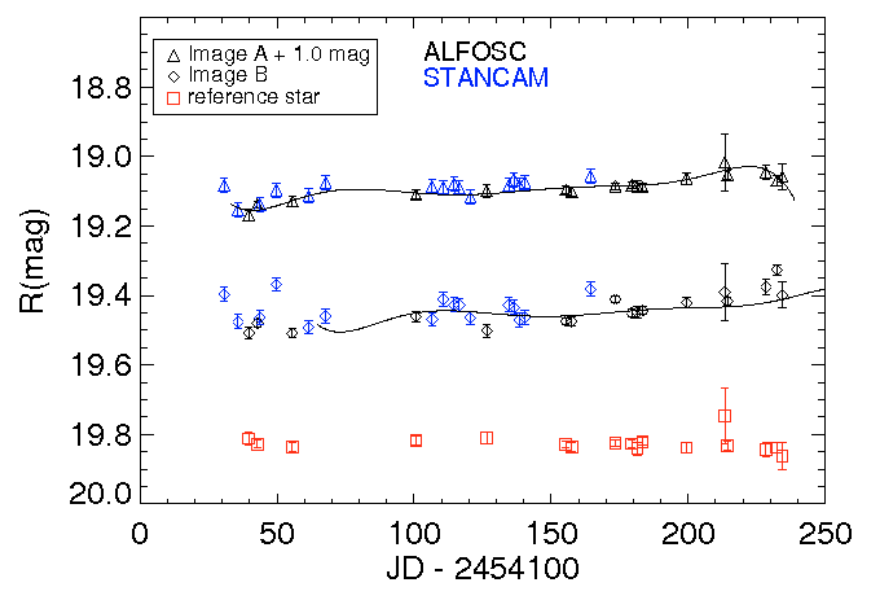

Fig. 5. $R$-band light curves of SDSS J1335+0118. A (diamonds) and B (triangles) images of the quasar are plotted with black and blue colors indicating weather data coming from ALFOSC or StanCam. The reference star is plotted with red squares. A polynomial is fitted to the A counterpart of the quasars light curve. This polynomial is also fitted to the B image with magnitude and time shift. The time shift is taken from the theoretical prediction of the time delay of this system (see Table 5).

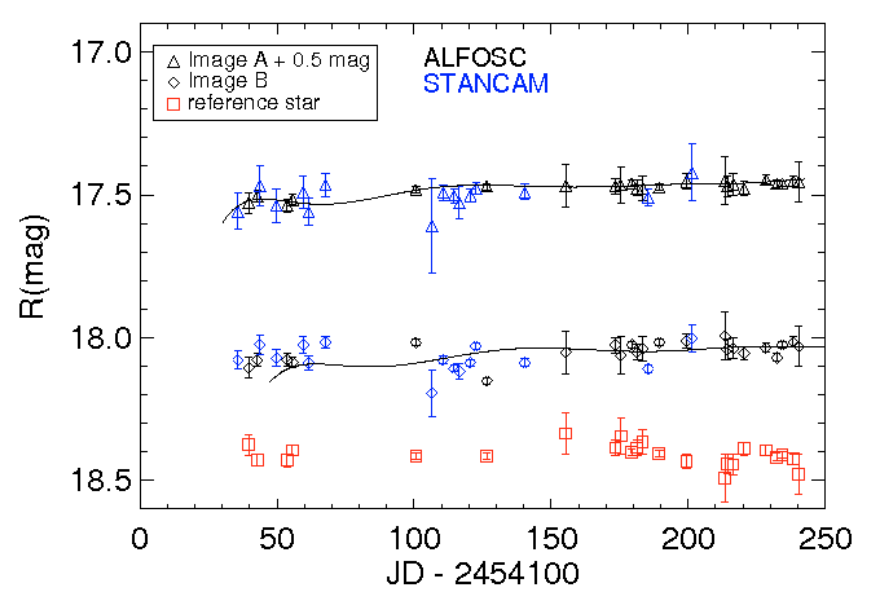

Fig. 6. $R$-band light curves of SDSS J1353+1138. Shapes and colors of the data point and fitting procedure identical with previous light curve. The time shift is taken from the theoretical prediction of the time delay of those systems (see Table 6).

no visible peaks in either light curves, the magnitude shift was chosen simply by taking a difference between average magnitude of image A and image B. The time shift is taken from the theoretical prediction of the time delay of those systems (see Sect. 8). The fitted polynomials do not show any preferred time shift. This means that due to the lack of quasar variability during the time span of the monitoring we did not manage to measure time delays in these systems.

\subsection{Quasars with variability}

In 2 out of the 5 monitored gravitationally lensed quasars variability was detected. In Figs. 7 and 8 we show the light curves of SDSS J1001+5027 and SDSS J1206+4332, respectively. 


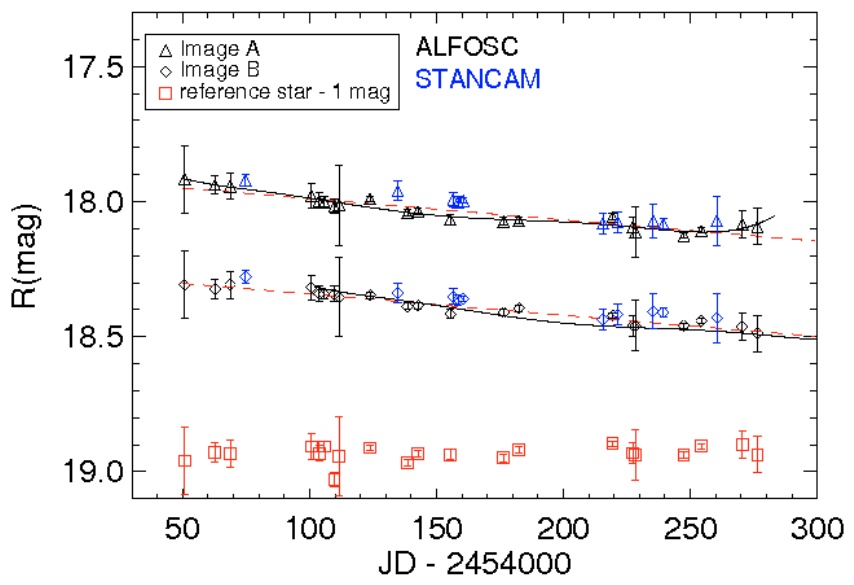

Fig. 7. $R$-band light curves of SDSS J1001+5027. Polynomials (solid, black lines) and linear regressions (dashed, red lines) are fitted to the A and B counterpart of the quasars separately. Shapes and colors of data point identical as described on previous plots.

\subsubsection{SDSS J1001+5027}

Figure 7 shows the light curves of SDSS J1001+5027 quasar images. We see that both images have some small variabilities in the light curve and also that both the $\mathrm{A}$ and $\mathrm{B}$ images have steadily decreased their brightness.

We have fitted a 7 th order polynomial (see Fig. 7 - black solid line) to the A data set. This was then fitted to the B light curve with magnitude shift which is the average magnitude difference between light curves $(0.4 \mathrm{mag})$ and time shift which is the predicted time delay ( 52 days).

We also have fitted a straight line to light curves (see Fig. 7 red dashed lines). The linear regression shows that both light curves have very similar evolution. Both images decreased their brightness by $0.2 \mathrm{mag}$ during the first 200 days. This indicates that the brightness decrease is due to long intrinsic quasar variability (de Vries et al. 2003).

Although variability is clearly visible, it is impossible to measure the time delay for this system just from the slope. The small fluctuations on the slope also do not give any conclusive results, neither visual shifting nor polynomial fitting help to find the time delay.

\subsubsection{SDSS J1206+4332}

In Fig. 8 we show the light curve of SDSS J1206+4332. We see clear long variabilities for both the A and B light curves. The variabilities are 100-150 days long, so the gaps in the sampling do not strongly influence the precision of the time delay estimation. The observed variabilities, consisting of bumps, allow for measurement of the time delay for SDSS J1206+4332, as detailed in Sect. 6.

\section{Time delay of SDSS J1206+4332}

From the geometry of the system SDSS J1206+4332 (see Fig. 3) we anticipate that the A image is leading since it is farther from the centre of the main lensing galaxy. Thus, we predict that any intrinsic quasar variabilities should appear first in the A image. From the mass modeling we know also that the time delay for this system is $\geq 50$ days (see Sect. 8). We apply these constraints when measuring the time delay from the light curves.

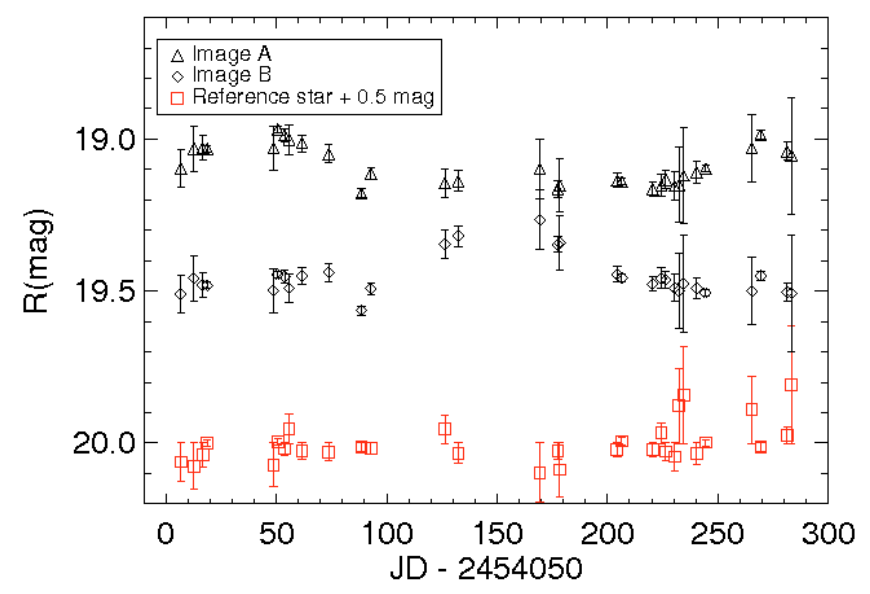

Fig. 8. $R$-band light curves of SDSS J1206+4332. Magnitude variabilities are seen in both light curves A (diamonds) and B (triangles) as bumps. The reference star is plotted with red squares.

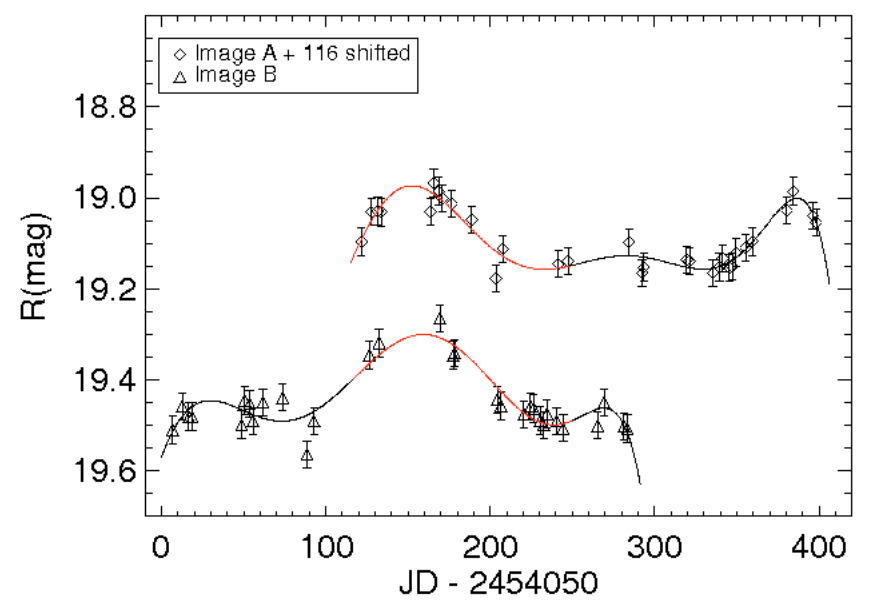

Fig. 9. $R$-band light curves of SDSS J1206+4332. Magnitude variabilities are seen in both light curves A (diamonds) and B (triangles) as bumps. The A light curve is shifted in time by 115 days. The red line indicates the intervals used for cross correlation.

The simplest form of such a measurement is visual shifting. Knowing that the variability has to appear first in the image A and then at least 50 days later in the image B we can associate the bump at the beginning of the A light curve centreed at $\sim 50$ days with the bump in the B light curve centreed at $\sim 150$ days.

A more quantitative measurement of a time delay is obtained by polynomial fitting. We fitted 7 th order polynomials to both light curves using minimum $\chi^{2}$. In Fig. 9 we show the two polynomials (solid lines). We have marked in red the parts of the polynomials which were used for calculating the time delay. In order to calculate the time delay we have shifted the A polynomial in time and magnitude to fit it to the $B$ data points and the B polynomial was shifted in time and magnitude to fit it to A data points. For each shift of the polynomials the goodness of fit was calculated. The results of these fits are presented in Fig. 10. For magnitude shift, $B-A=0.31$ mag the average of the two time delay estimations is $116_{-7}^{+6}$ days, where errors are uncertainties of the two values of the time delay combined in quadrature. Based on our modeling (G1 - SIE and G3 - SIS) (see Sect. 8) and 


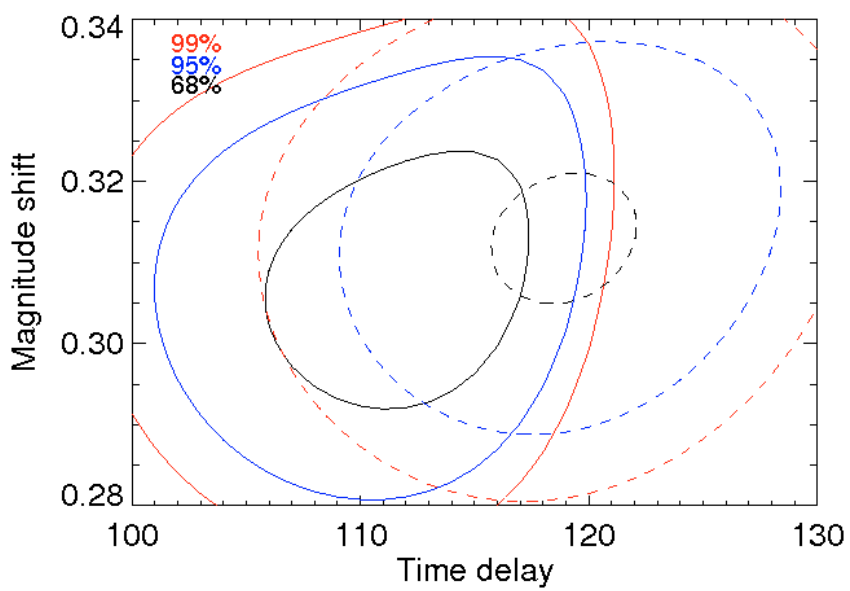

Fig. 10. Results of cross correlation between data on one image and fitted polynomial fitted to light curve of second image. Solid lines represents cross correlation between the A data and the polynomial fitted to $\mathrm{B}$ data; dashed lines are representing cross correlation between the $\mathrm{B}$ data and a polynomial fitted to the A data.

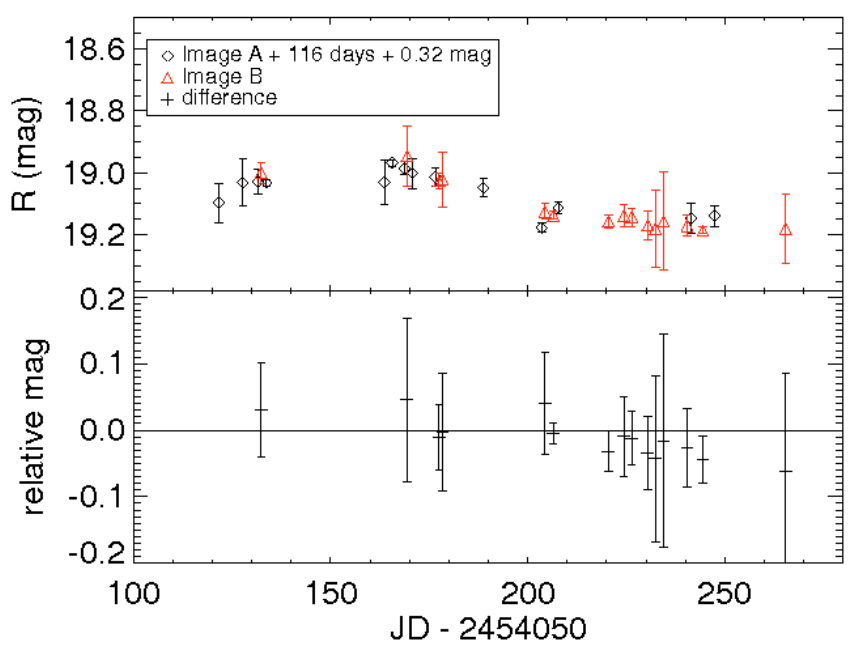

Fig. 11. Microlensing of SDSS J1206+4332. Top: time-delay shifted light curves, with the A image offset by 0.32 mag and 115 days. Bottom: difference between linearly interpolated A image and B image.

using the measured time delay ( $\Delta \tau=116_{-7}^{+6}$ days) we find a Hubble constant of $73_{-4}^{+3} \mathrm{~km} \mathrm{~s}^{-1} \mathrm{Mpc}^{-1}$.

\section{Microlensing}

Chang \& Refsdal (1979) predicted that in lensed quasar systems the light path should be affected by stars in the lensing galaxy. Moving compact objects in the lensing galaxy can cause spectral changes, brightness variability and, in the case of multiple images, flux-ratio anomalies in the lensed quasar.

The images of a lensed quasar may vary due to intrinsic quasar brightness changes and/or microlensing. Microlensing affects the light paths of each image differently (in the simplest case only one path is affected) whereas the intrinsic variations show up in all the images but at different times due to the time delay. Therefore, one can isolate the microlensing signal by simply calculating the difference between two light curves.

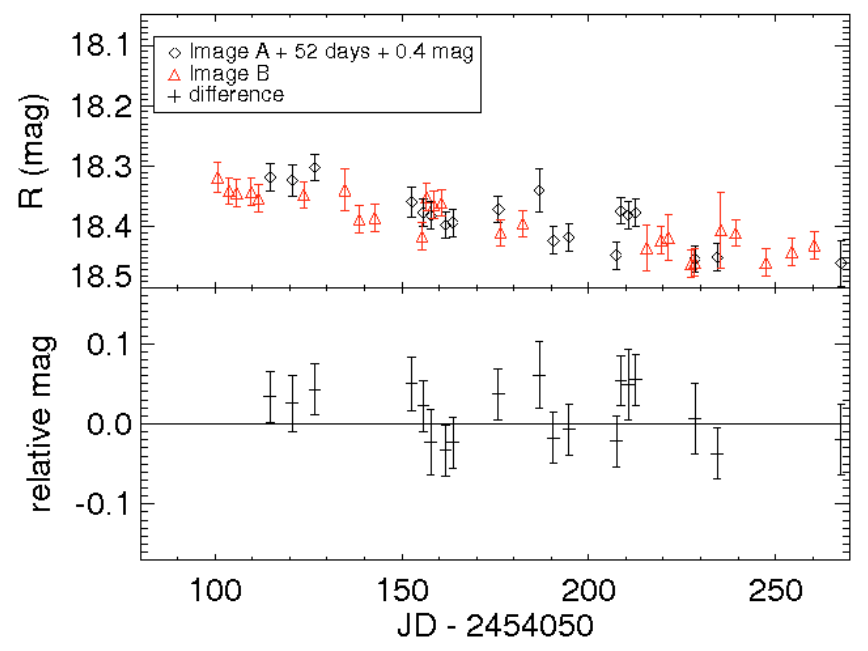

Fig. 12. Microlensing of SDSS J1001+5027. Top: time-delay shifted light curves, with the A image offset by 0.4 mag and 52 days. Bottom: difference between linearly interpolated A image and B image.

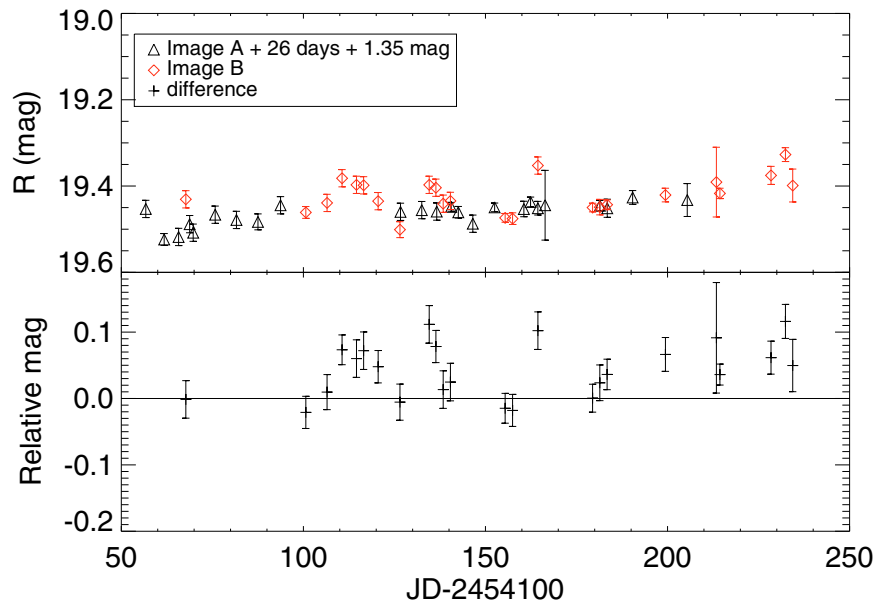

Fig. 13. Microlensing of SDSS J1335+0118. Top: time-delay shifted light curves, with the A image offset by 1.35 mag and 26 days. Bottom: difference between linearly interpolated A image and B image.

If we shift one of the light curves in magnitude (by the magnitude difference between light curves) and in time (by the time delay) and subtract it from the other quasar counterpart light curve the remaining variation in the light curve difference should not belong intrinsically to the quasar but rather to microlensing.

Our previous study (Paraficz et al. 2006) showed that microlensing variabiliy can be detected in this way. The monitoring program presented in this paper was designed to be sensitive to such microlensing signals. The only limitation are $\mathrm{S} / \mathrm{N}$ and gaps in the light curve due to bad weather conditions. In Figs. 11-15 we present the result of a microlensing variability analysis for our observed targets.

The top plots present the quasar image light curves, with one of the images being shifted in time (by the theoretically predicted or, if available, the measured time delay) and in magnitude (by the average magnitude difference between the images). The lower panels show the light curve differences. The difference is calculated between a linearly interpolated B light curve and the A data points. In Figs. 11-15 we see that there is no 


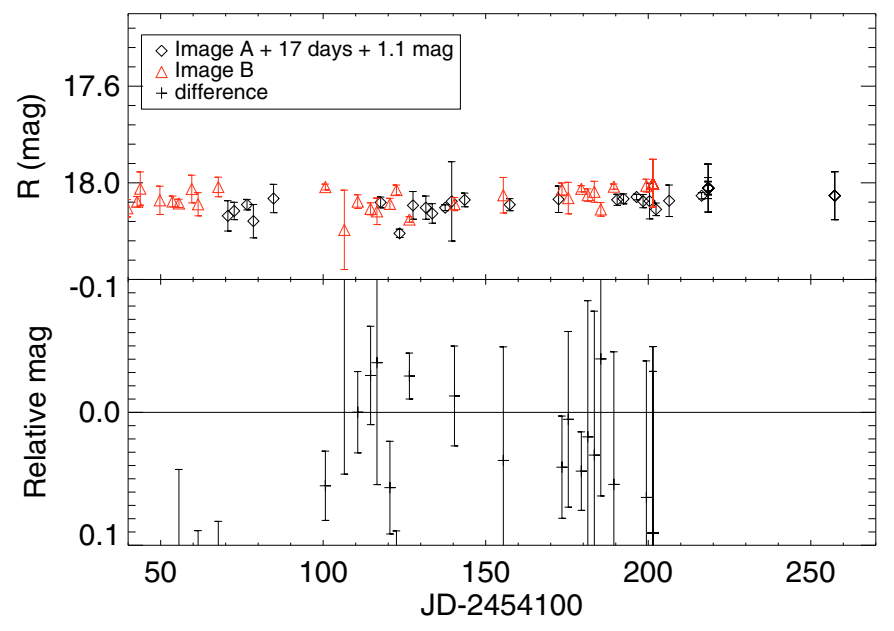

Fig. 14. Microlensing of SDSS J1353+1138. Top: time-delay shifted light curves, with the A image offset by $1.1 \mathrm{mag}$ and 17 days. Bottom: difference between linearly interpolated A image and B image.

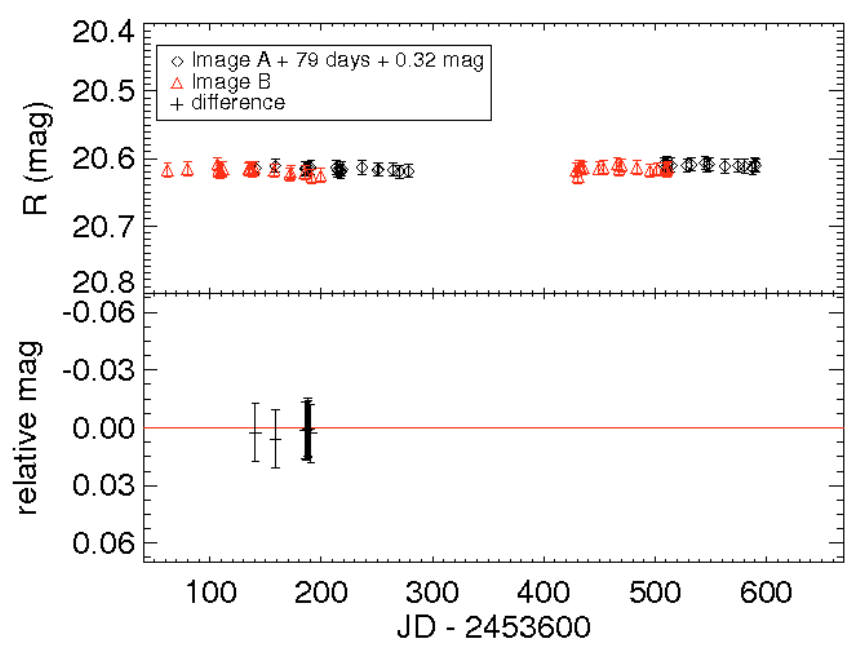

Fig. 15. Microlensing of SDSS J0903+5028. Top: time-delay shifted light curves, with the A image offset by 0.32 mag and 79 days. Bottom: difference between linearly interpolated A image and B image.

signal stronger than 0.1 mag which is $\sim 2 \sigma$. We therefore conclude that we did not detect any significant microlensing event in any of the systems during our monitoring program.

\section{Modeling}

\subsection{Analytical modeling}

In principle, one can calculate the Hubble constant from the time delay of a gravitationally lensed system, but there is one major obstacle, namely the mass-sheet degeneracy (Falco et al. 1985). This degeneracy is between the Hubble constant and the radial density profiles of the lens (Wucknitz \& Refsdal 2001) and its angular structure (Freedman et al. 2001). Summarizing, different mass profiles give different results on the Hubble constant without changing the lensing configuration. To break this degeneracy we need to have information about the mass profile, e.g., through the velocity dispersion (Romanowsky \& Kochanek 1999). For our systems we do not know which lens model is the correct one, but fortunately in many cases a lens can be quite well modeled using a fairly simple mass model like SIE (Singular Isothermal Ellipsoid) (Kormann et al. 1994) or NFW (Navarro-Frank-White) (Golse \& Kneib 2002).

In this paper, for the analytical modeling, we have modeled all our systems using singular isothermal potentials. We used those models because of their simplicity and because they agree with the physical properties of many observed lenses (Kochanek 1993; Rusin et al. 2003; Koopmans et al. 2006).

In the analytical modeling we have the positions of the two images as constraints and as free parameters we have the lens velocity dispersion, $\sigma_{0}$ and the ellipticity, $\epsilon$. We also have two fixed parameters, the position angle, $\theta_{\epsilon}$ and the position of the lens galaxy. The number of degrees of freedom is 0 , thus we can look for the one model which perfectly fits the data. The positions of the images and lens are visible on the decovolved images (see Fig. 3). The position angle of the lenses can be roughly estimated from analysis of the deconvoled images, as the mass profile of a lens tends to align to its visible component (Keeton et al. 1998). We allow this constraint to vary within $45^{\circ}$. We have not used flux ratios as constraints because of the possible influence of reddening by dust (Elíasdóttir et al. 2006), microlensing (Paraficz et al. 2006) or small-scale structure in the lens potential (Dalal \& Kochanek 2002).

The ellipticity used here is defined as $\epsilon=\left(a^{2}-b^{2}\right) /\left(a^{2}+b^{2}\right)$, where $a$ and $b$ are the major and minor axis. The position angle corresponds to the direction of the semi-major axis of the isopotential counted east of north. The modeling has been performed using the LENSTOOL software package available at http : //www . oamp . fr/cosmology/lenstool/ (Jullo et al. 2007). LENSTOOL is a software created for modelling strong lensing systems with parametric methods which chooses models using the Bayesian evidence. In all models we set the Hubble constant to be $H_{0}=73 \mathrm{~km} \mathrm{~s}^{-1} \mathrm{Mpc}^{-1}$.

\subsubsection{SDSS J0903+5028}

We have fitted the two simple models, SIS (Singular Isothermal Sphere) and SIE (Singular Isothermal Ellipsoid) to the lensing galaxy of SDSS J0903+5028. We have assumed that only one galaxy, the central one, influences the quasar light, even though other galaxies are visible to the north and south of the images (see Fig. 16). The lensing images of the quasar are not collinear with the central galaxy which indicates that there is a quadruple moment in the potential. This moment can come from the tidal effect of the nearby galaxies or may be due to elongation of the central galaxy. Because of the degeneracy we are not able to distinguish which one of the possibilities is true, thus we use one lens, an SIE model which accurately predicts image positions. In Table 2 we summarize the results of the SDSS J0903+5028 modeling where the central single lens is an SIE. Based on this model, the predicted time delay for the system is 79.4 days. The uncertainty in this value is entirely dominated by systematic modeling uncertainties.

\subsubsection{SDSS $J 1001+5027$}

The lens of SDSS J1001+5027 consists of two galaxies (see Fig. 3) which according Oguri et al. (2005) are at similar redshifts $0.2<z<0.5$. For our modeling we fix both lensing galaxies at redshift 0.3 .

We have created two possible models of the lens environment of SDSS J1001+5027. In the first one the system has only one galaxy with elongated SIE mass profile. This model is able 


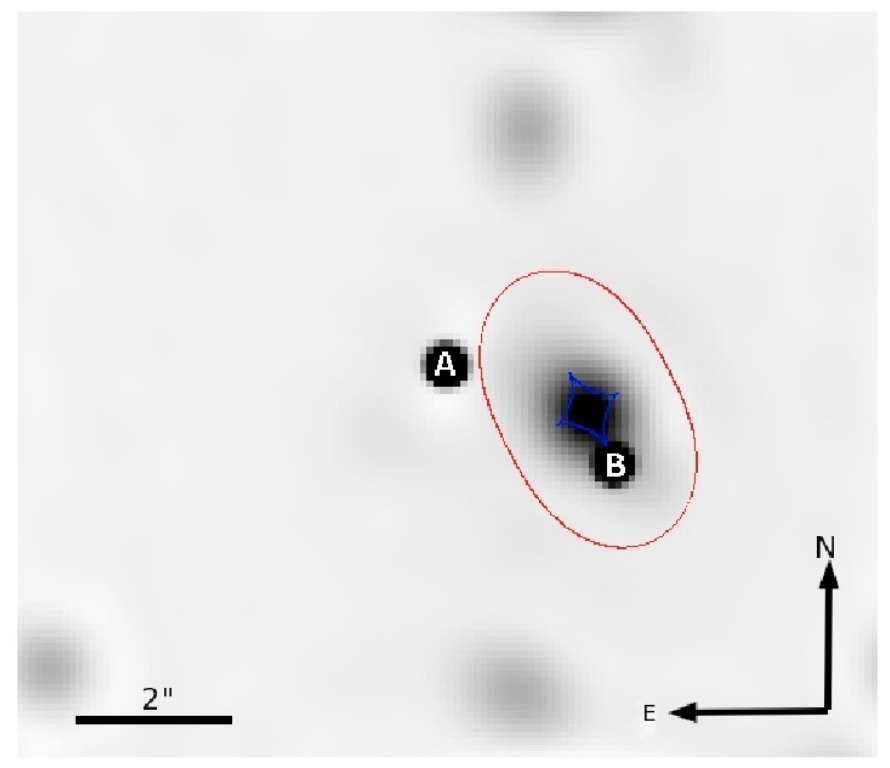

Fig. 16. Lens model of SDSS J0903+5028. The figure shows an image of the system with over-plotted results of lens modeling. Blue and red lines represent critical and caustic lines, respectively.

Table 2. Lens model of SDSS J0903 $+5028^{2}$.

\begin{tabular}{cccccccc}
\hline \hline$\#$ & Type & $\begin{array}{c}x \\
(\operatorname{arcsec})\end{array}$ & $\begin{array}{c}y \\
(\operatorname{arcsec})\end{array}$ & $\begin{array}{c}\sigma_{0} \\
\left(\mathrm{~km} \mathrm{~s}^{-1}\right)\end{array}$ & $\epsilon$ & $\theta_{\epsilon}$ & $\begin{array}{c}\Delta \tau \\
(\text { days })\end{array}$ \\
\hline $\mathrm{G}$ & SIE & 1.99 & -0.70 & 264.6 & 0.49 & $27^{\circ}$ & 79.4 \\
\hline
\end{tabular}

${ }^{2}$ Modeled parameters are galaxy position, $x, y$, lens velocity dispersion, $\sigma_{0}$, ellipticity, $\epsilon$, position angle, $\theta_{\epsilon}$ and time delay, $\Delta \tau$.

to ray-trace the position of the quasar images. From Fig. 17 we also see that the position angle, PA of the model is consistent with the light distribution of the main galaxy but it is also in the direction of the other galaxy. This leads to the conclusion that the quadruple moment might come from the second galaxy rather then from the elongation of the main one. In the second version of the lens model we have set both gravitational lenses as Singular Isothermal Spheres, SIS. This model also reproduces the position of the quasar images and gives a sensible result of the galaxy velocity dispersions being $223 \mathrm{~km} \mathrm{~s}^{-1}$ for main lens (G1) and $159 \mathrm{~km} \mathrm{~s}^{-1}$ for G2. The results from both models are presented in Fig. 17 and summarized in Table 3. The predicted time delay of the system is 34 days based on the SIE model and 52 days based on the double SIS model.

\subsubsection{SDSS J1206+4332}

We have fitted SIS and SIE to the main lensing galaxy of the SDSS J1206+4332. We have assumed that the only galaxy which influences the quasar light is the central galaxy G1 (see Fig. 3). Unfortunately we come to the same conclusions as (Oguri et al. 2005) that neither of these mass models gives physically realistic results. The system seems to be more complicated, so presumably the other galaxies seen in vicinity of the system may play a role. A model of the main galaxy G1 (SIE) and G2 (SIS) does not give any physical solutions either, which is expected since the redshift of $\mathrm{G} 2$ is predicted to be rather small.

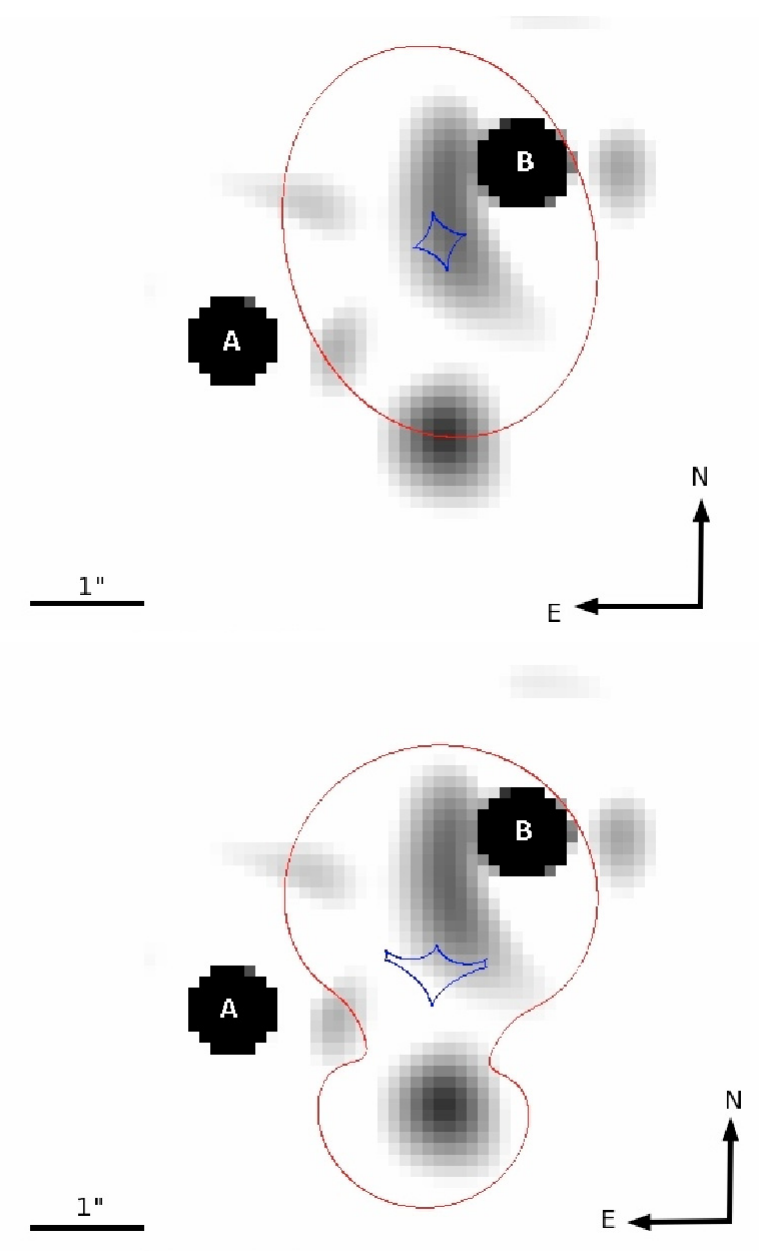

Fig. 17. Lens model of SDSS J1001+5027. The figure shows an image of the system with over-plotted results of lens modeling. Blue and red lines represent critical and caustic lines, respectively. Top: plot present the single SIE lens model and bottom: plot presents the two SIS lens models.

Table 3. Lens model of SDSS J1001+5027³.

\begin{tabular}{cccccccc}
\hline \hline$\#$ & Type & $\begin{array}{c}x \\
(\operatorname{arcsec})\end{array}$ & $\begin{array}{c}y \\
(\operatorname{arcsec})\end{array}$ & $\begin{array}{c}\sigma_{0} \\
\left(\mathrm{~km} \mathrm{~s}^{-1}\right)\end{array}$ & $\epsilon$ & $\theta_{\epsilon}$ & $\begin{array}{c}\Delta \tau \\
(\text { days })\end{array}$ \\
\hline G1 & SIE & 1.749 & 0.861 & 258 & 0.23 & $11^{\circ}$ & 52 \\
\hline G1 & SIS & 1.749 & 0.861 & 223 & 0 & $\cdots$ & 34 \\
G2 & SIS & 1.629 & -0.588 & 159 & 0 & $\cdots$ & 34 \\
\hline
\end{tabular}

${ }^{3}$ Column headings: cf. Table 2.

It appears that the third galaxy lying $\sim 3^{\prime \prime}$ north of the system (see Fig. 3) has a strong influence on the system geometry. If we assume our model to be two lensing galaxies with one of them being G1 (SIE) and the other G3 (SIS) it is possible to reproduce the positions of the images. The results of that final fitting are summarized in Table 4. Based on this model, the predicted time delay of the system is 112.5 days which is in agreement with our measurement of $116_{-7}^{+6}$ days (see Sect. 6).

\subsubsection{SDSS J1335+0118}

The system has one lensing galaxy, at redshift $z=0.5$, which we have modeled as an SIE. The lensing galaxy is barely visible in our images, hence the position of the galaxy was taken from Oguri et al. (2004). According to observations made by 


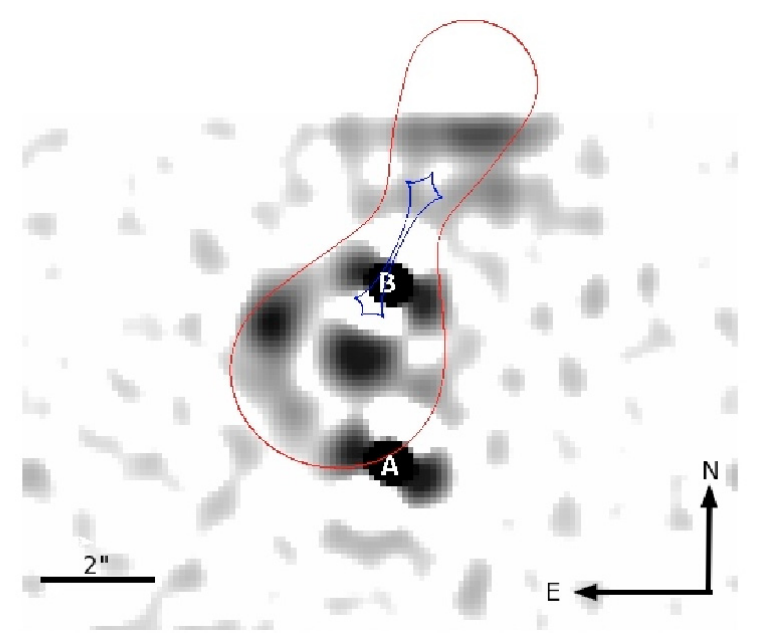

Fig. 18. Lens model of SDSS J1206+4332. The figure shows an image of the system with over-plotted results of lens modeling. Blue and red lines represent critical and caustic lines respectively.

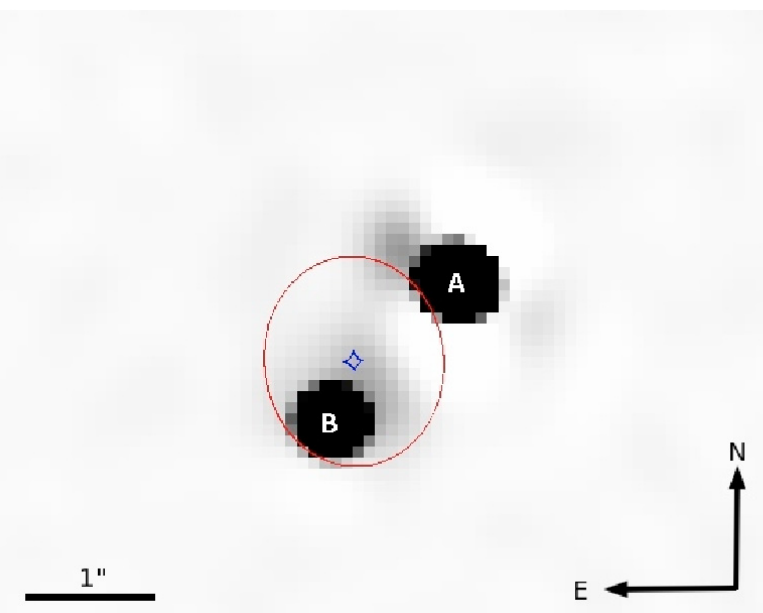

Fig. 19. Mass model of SDSS J1335+0118. Figure shows an image of the system with over-plotted results of lens modeling. Blue and red lines represent critical and caustic lines, respectively.

Table 4. Lens model of SDSS J1206+4332 ${ }^{3}$.

\begin{tabular}{cccccccc}
\hline \hline$\#$ & Type & $\begin{array}{c}x \\
(\operatorname{arcsec})\end{array}$ & $\begin{array}{c}y \\
(\operatorname{arcsec})\end{array}$ & $\begin{array}{c}\sigma_{0} \\
\left(\mathrm{~km} \mathrm{~s}^{-1}\right)\end{array}$ & $\epsilon$ & $\theta_{\epsilon}$ & $\begin{array}{c}\Delta \tau \\
(\text { days })\end{array}$ \\
\hline G1 & SIE & -0.66 & 1.75 & 347.5 & 0.1 & $-2^{\circ}$ & 112.5 \\
G3 & SIS & 1.32 & 5.9 & 279.5 & 0 & $\cdots$ & 112.5 \\
\hline
\end{tabular}

${ }^{3}$ Column headings: cf. Table 2 .

Oguri et al. (2004) with Keck, the lensing galaxy is misaligned with the QSO images which indicates the existence of external shear or elongation of the lensing galaxy. A model with an SIE lens accurately predicts the image position and gives plausible galaxy parameters. The model details are summarized in Table 5. The estimated time delay of the system is 26.2 days which is in agreement with previous studies.

\subsubsection{SDSS J1353+1138}

SDSS J1353+1138 has a centrally aligned lensing galaxy and the B image very close to the galaxy centre. There are no other

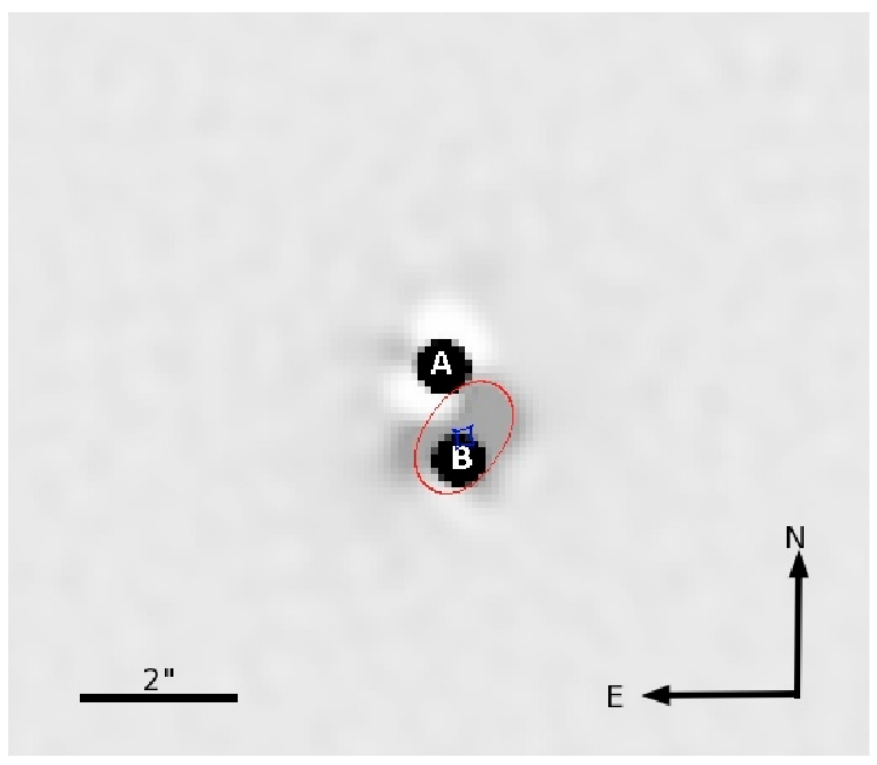

Fig. 20. Mass model of SDSS J1353+1138. Figure shows an image of the system with over-plotted results of lens modeling. Blue and red lines represent critical and caustic lines, respectively.

Table 5. Lens model of SDSS J1335+0118 ${ }^{3}$.

\begin{tabular}{cccccccc}
\hline \hline$\#$ & Type & $\begin{array}{c}x \\
(\operatorname{arcsec})\end{array}$ & $\begin{array}{c}y \\
(\operatorname{arcsec})\end{array}$ & $\begin{array}{c}\sigma_{0} \\
\left(\mathrm{~km} \mathrm{~s}^{-1}\right)\end{array}$ & $\epsilon$ & $\theta_{\epsilon}$ & $\begin{array}{c}\Delta \tau \\
(\text { days })\end{array}$ \\
\hline $\mathrm{G}$ & SIE & -0.786 & -0.649 & 218 & 0.16 & $-5^{\circ}$ & 26.2 \\
\hline
\end{tabular}

${ }^{3}$ Column headings: cf. Table 2.

Table 6. Lens model of SDSS J1353+1138 ${ }^{3}$.

\begin{tabular}{cccccccc}
\hline \hline$\#$ & Type & $\begin{array}{c}x \\
(\operatorname{arcsec})\end{array}$ & $\begin{array}{c}y \\
(\operatorname{arcsec})\end{array}$ & $\begin{array}{c}\sigma_{0} \\
\left(\mathrm{~km} \mathrm{~s}^{-1}\right)\end{array}$ & $\epsilon$ & $\theta_{\epsilon}$ & $\begin{array}{c}\Delta \tau \\
(\text { days })\end{array}$ \\
\hline $\mathrm{G}$ & SIE & -0.25 & -1.04 & 177 & 0.46 & $35^{\circ}$ & 17.2 \\
\hline
\end{tabular}

${ }^{3}$ Column headings: cf. Table 2.

close-by galaxies; thus the lens can be modeled with a simple SIE. The predicted time delay for that system is 17 days (see Table 6) which is in agreement with previous studies.

\subsection{Non-parametric modeling}

We have also modeled our systems based on a non-parametric method proposed by Saha \& Williams (1997) using the publicly available code PixeLens (Saha \& Williams 2004).

PixeLens reconstructs a pixelated mass map of the lens by implementing Bayesian statistics. It generates numerous lens models which fit the lens system geometry. There are two major advantages of using this method. First, it allows to explore a wide range of models not restricted with parameters, which circumvents the non-uniqueness problem of lens modeling. Second, it provides an estimate of the systematic errors on the modeled time delay which is not possible to get when using analytical methods. Our purpose of using it is to check the robustness of our time delay estimations, i.e. we want to know whether the two approaches, analytical and pixeleted, give consistent results for the time delays. PixeLens generates models which reproduce the exact position of lensing images and aligned within $45^{\circ}$ of the visible lens PA. All our systems were modeled assuming existence of external shear and all of them, 
Table 7. Modeled time delays of the five lensed quasars.

\begin{tabular}{cccc}
\hline \hline$\#$ & $\begin{array}{c}\text { Analytical } \\
\text { (days) }\end{array}$ & $\begin{array}{c}\text { PixeLens (average) } \\
\text { (days) }\end{array}$ & $\begin{array}{c}\text { PixeLens (median) } \\
\text { (days) }\end{array}$ \\
\hline $0903+5028$ & 79.4 & $98 \pm 21$ & $101_{-12}^{+9}$ \\
$1001+5027$ & 52 & $81 \pm 23$ & $76_{-9}^{+14}$ \\
$1206+4332$ & 113 & $173 \pm 64$ & $152_{-21}^{+41}$ \\
$1335+0118$ & 26 & $32 \pm 8$ & $35_{-6}^{+2}$ \\
$1353+1138$ & 17 & $21 \pm 5$ & $20_{-2}^{+3}$ \\
\hline
\end{tabular}

except SDSS J1206+4332, were modeled as symmetric. For each system we generated 300 lens models which were used to estimate the average and median time delay and their model uncertainties. The modeling results of the 5 gravitationally lensed QSOs with PixeLens are summarized in Table 7. Figure 21 shows the potentials and time delays of the 5 systems modeled with pixelated method. On the left side we see potential contours modeled by PixeLens which are over-plotted on the mass map created in analytical modeling. On the right are histograms of the time delays generated for each system by the 300 lens models.

We see that the lens potentials of the PixeLens correspond to the analytical models and from the Table 7 we see that the estimated time delay values from analytical modeling lie within error bars of the PixeLens time delays. This is reassuring and gives confidence in our approach. We note also that the analytically obtained time delays are systematically lower than the average values obtained using PixeLens by $20-50 \%$.

\section{Simultanous modeling}

The Hubble constant estimated from time delay measurements is model dependent, as illustrated above. Different models give a different Hubble constant and measuring the time delay with high precision does not resolve that issue. One way of dealing with this problem is to generate a large number of models of many lenses and create a distribution of possible values for the Hubble constant based on these models. Thus, we use the pixelated method for several gravitationally lensed systems constraining them to have the same shared value of $H_{0}$. Simultaneous modeling has been done in the past by e.g. Saha et al. (2006) - with 10 lenses and Coles (2008) - with 11 lenses.

Figure 22 shows the result of the simultaneous modeling of 5 systems with time delays obtained at the NOT. These are RX J0911+055 (Hjorth et al. 2002), SBS B1520+530, (Burud et al. 2002b), B1600+434 (Burud et al. 2000) and J0951+263 (Jakobsson et al. 2005) and the time delay of SDSS J1206+4332 reported in this paper. On the histogram the results of our simultaneous modeling of the 5 QSOs are presented. For each object 100 lens models were created. The Hubble constant estimated using this method is $H_{0}=61.5_{-4}^{+8} \mathrm{~km} \mathrm{~s}^{-1} \mathrm{Mpc}^{-1}$.

\section{Discussion}

We have estimated the time delay of the SDSS J1206+4332 to be $\Delta \tau=116_{-7}^{+6}$ based on a 280 -day long monitoring campaign at the Nordic Optical Telescope. This shows the feasibility of measuring time delays from campaigns not much longer than the time delay itself. To model the system, we first assumed a single lensing galaxy modeled with the Singular Isothermal
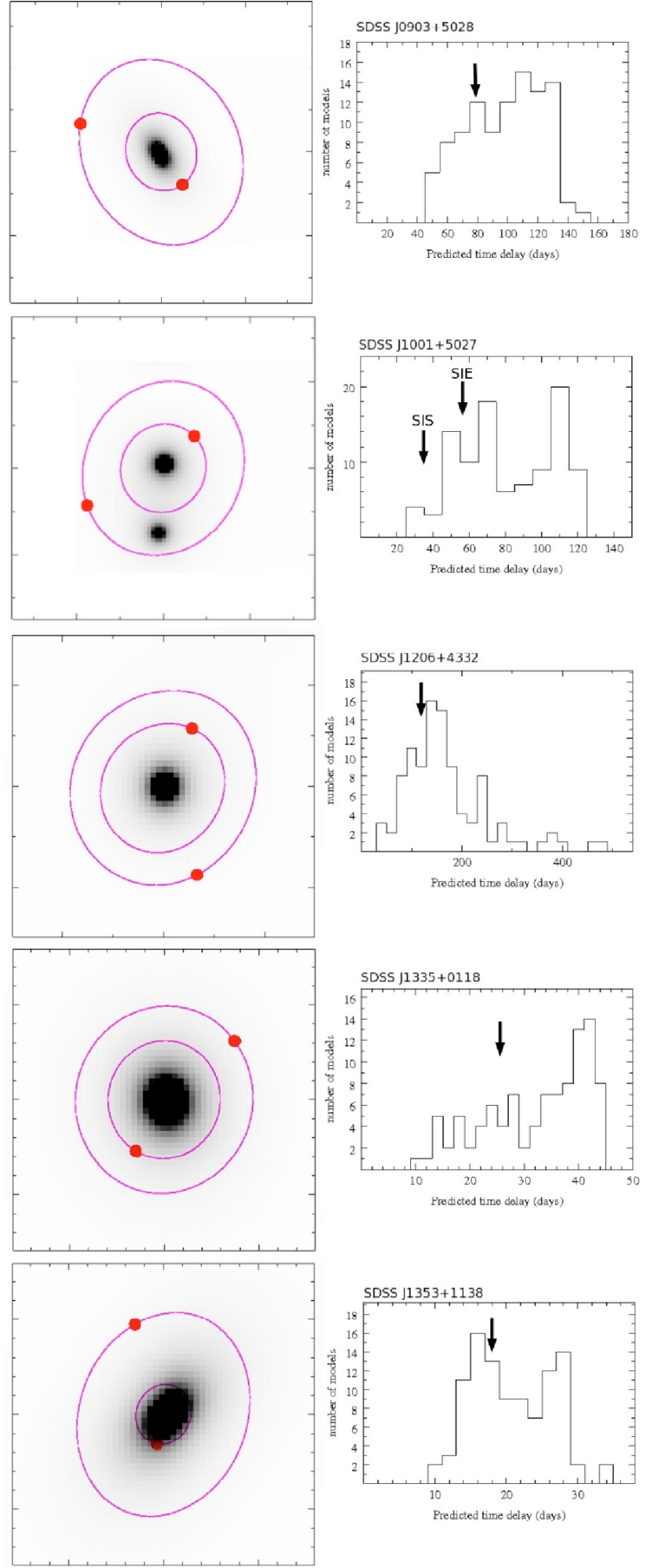

Fig. 21. The modeling results of the five lensed quasars. On the left side we see potential contours modeled by PixeLens which are over-plotted on the mass map created in analytical modeling. On the right the are time delay histograms generated for each system by the 300 suitable lens models, arrows indicate the time delays estimated from analytical modeling. We see that the potentials of the PixeLens correspond quite well to the analytical models. 
Hubble constant (local units)

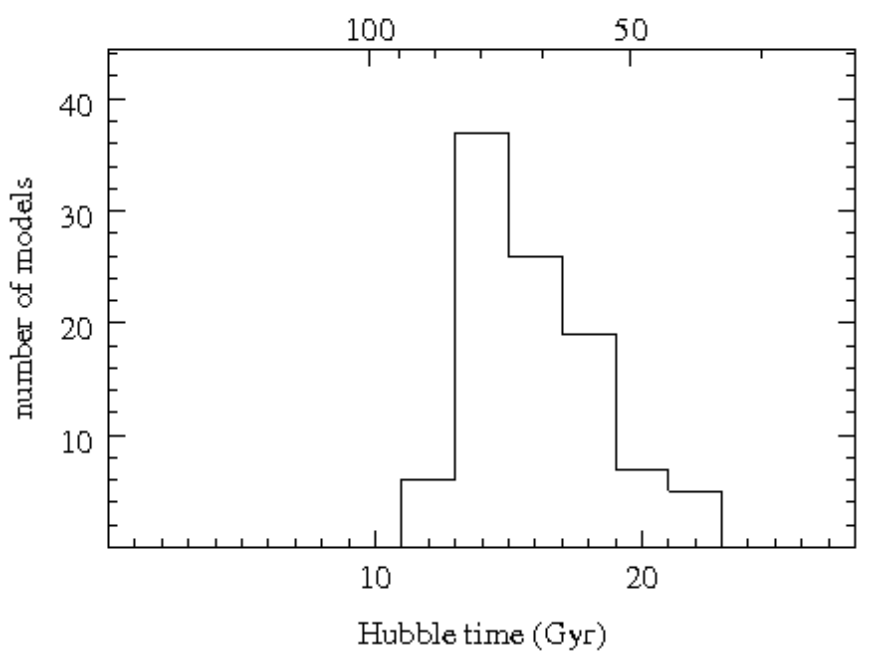

Fig. 22. The result of the simultaneous modeling. It is a histogram created from the modeling of the 5 QSOs monitored at the NOT for each of them 100 lens models were created, the Hubble constant estimated using this method is $H_{0}=61.5_{-4}^{+8} \mathrm{~km} \mathrm{~s}^{-1} \mathrm{Mpc}^{-1}$.

Ellipsoid (SIE), but were unable to reproduce the image positions. By adding to the lens model the $\mathrm{G} 2$ and $\mathrm{G} 3$ galaxies we found that the influence of the G2 galaxy is negligible due to its small redshift but the G3 galaxy has a major impact on the system's geometry. A lens model with two galaxies, where the main one is a SIE and the second is a SIS, predicts the image positions very well. Thus this model resulted in a Hubble constant of $73_{-4}^{+3} \mathrm{~km} \mathrm{~s}^{-1} \mathrm{Mpc}^{-1}$ assuming $\Omega_{\mathrm{m}}=0.3$ and $\Omega_{\Lambda}=0.7$.

Lack of short-term variability in the other monitored systems (SDSS J0903, SDSS J1001+5027, SDSS J1353+1138, SDSS J1335+0118) meant that we did not succeed in measuring their time delays. From the quasar studies of Fohlmeister et al. (2008), Vuissoz et al. (2007), Koopmans et al. (2000) and many others we see that during the 200-300 days of a quasar monitoring a quasar might be at the quiet stage or at the slow increase or decrease. Thus, despite our success in measuring the time delay of SDSS J1206+4332, it appears that monitoring substantially longer than the predicted time delay of a given system will be unavoidable in many cases.

We have modeled all five systems using both analytical and pixelated methods. Using the predicted time delays we have shown that none of the systems exhibited significant microlensing variability during the observing campaign.

We have also performed simultaneous pixelated modeling with a common Hubble constant of five lenses for which time delays were measured at the NOT. The estimated Hubble constant from this analysis is $H_{0}=61.5_{-4}^{+8} \mathrm{~km} \mathrm{~s}^{-1} \mathrm{Mpc}^{-1}$.

Acknowledgements. The Dark Cosmology Centre is funded by the DNRF. D.P. acknowledges receipt of a research studentship at the Nordic Optical Telescope. Á.E. acknowledges the support of the EU under a Marie Curie International Outgoing Fellowship, contract PIOF-GA-2008-220049. We thank Andreas Jaunsen for his contribution in the definition phase of the project, Jin Hyeok An and Andrea Morandi for useful comments and Páll Jakobsson for extensive help with MCS deconvolution. Also, we would like to thank all visiting observers at the NOT who obtained data on our behalf. The data used are based on observations made with the Nordic Optical Telescope, operated on the island of La Palma jointly by Denmark, Finland, Iceland, Norway, and Sweden, in the Spanish Observatorio del Roque de los Muchachos of the Instituto de Astrofisica de Canarias.

\section{Appendix A: Photometry}

Table A.1. Photometry of two images of SDSS J0903+5028 quasar.

\begin{tabular}{ccc}
\hline \hline $\begin{array}{c}\mathrm{A} \\
(\mathrm{mag})\end{array}$ & $\begin{array}{c}\mathrm{B} \\
(\mathrm{mag})\end{array}$ & $\begin{array}{c}\text { JD } \\
\text { (days) }\end{array}$ \\
\hline 20.30419 & 20.61679 & 61.732670 \\
20.30074 & 20.61527 & 79.761260 \\
20.30507 & 20.60809 & 106.76752 \\
20.30473 & 20.61668 & 107.72829 \\
20.30655 & 20.61970 & 108.77146 \\
20.30474 & 20.61881 & 109.75729 \\
20.30363 & 20.61556 & 111.70990 \\
20.30291 & 20.61626 & 134.49363 \\
20.30774 & 20.61529 & 135.60198 \\
20.30557 & 20.61790 & 137.56633 \\
20.30945 & 20.61812 & 138.65267 \\
20.30545 & 20.61433 & 139.65707 \\
20.30296 & 20.61801 & 157.56273 \\
20.30661 & 20.62338 & 171.54778 \\
20.30592 & 20.62005 & 172.58139 \\
20.30660 & 20.62089 & 185.40632 \\
20.30960 & 20.62697 & 191.46353 \\
20.30862 & 20.62502 & 199.52655 \\
20.29831 & 20.61721 & 428.69561 \\
20.30180 & 20.62714 & 430.74676 \\
20.29676 & 20.61238 & 431.69420 \\
20.29825 & 20.61207 & 432.73686 \\
20.29982 & 20.61431 & 435.74226 \\
20.30070 & 20.61477 & 449.59337 \\
20.29906 & 20.61360 & 453.71216 \\
20.29610 & 20.60806 & 465.70068 \\
20.30069 & 20.61581 & 467.67382 \\
20.29816 & 20.60991 & 469.74747 \\
20.30101 & 20.61356 & 483.76553 \\
20.30075 & 20.61761 & 495.56600 \\
20.30173 & 20.61585 & 501.74698 \\
20.30377 & 20.61614 & 508.74511 \\
\hline & & \\
& & \\
20
\end{tabular}

Table A.2. Photometry of two images of SDSS J1001+5027 quasar and a reference star.

\begin{tabular}{|c|c|c|c|c|}
\hline $\begin{array}{c}\mathrm{A} \\
(\mathrm{mag})\end{array}$ & $\begin{array}{c}\mathrm{B} \\
(\mathrm{mag})\end{array}$ & $\begin{array}{c}\text { Ref } \\
(\mathrm{mag})\end{array}$ & $\begin{array}{l}\text { Error } \\
(\mathrm{mag})\end{array}$ & $\begin{array}{c}\text { JD } \\
\text { (days) }\end{array}$ \\
\hline 18.30830 & 17.91642 & 19.95965 & 0.12438 & 50.69800 \\
\hline 18.32345 & 17.93772 & 19.92900 & 0.03465 & 62.73200 \\
\hline 18.30794 & 17.94314 & 19.93315 & 0.04938 & 68.69100 \\
\hline 18.27762 & 17.92192 & $\ldots$ & 0.02200 & 74.71047 \\
\hline 18.31762 & 17.97847 & 19.90674 & 0.04654 & 100.66100 \\
\hline 18.34080 & 17.99653 & 19.93185 & 0.02832 & 103.73100 \\
\hline 18.34324 & 18.00136 & 19.90626 & 0.01881 & 105.76800 \\
\hline 18.34226 & 18.01672 & 20.02877 & 0.02820 & 109.72400 \\
\hline 18.35312 & 18.01316 & 19.94301 & 0.14773 & 111.65300 \\
\hline 18.34697 & 17.99086 & 19.91171 & 0.01053 & 123.78600 \\
\hline 18.33916 & 17.95949 & $\cdots$ & 0.03468 & 134.75378 \\
\hline 18.38763 & 18.04212 & 19.96631 & 0.01190 & 138.58600 \\
\hline 18.38510 & 18.03704 & 19.93143 & 0.01279 & 142.76500 \\
\hline 18.41525 & 18.06692 & 19.93604 & 0.01870 & 155.43300 \\
\hline 18.35080 & 17.99321 & $\cdots$ & 0.02762 & 156.58644 \\
\hline 18.36362 & 18.00054 & $\cdots$ & 0.02009 & 158.59506 \\
\hline 18.36016 & 17.99648 & $\cdots$ & 0.01003 & 160.60051 \\
\hline 18.40997 & 18.07270 & 19.94891 & 0.01356 & 176.41200 \\
\hline 18.39495 & 18.07015 & 19.91818 & 0.01065 & 182.41100 \\
\hline 18.43531 & 18.07975 & $\cdots$ & 0.03784 & 215.53896 \\
\hline 18.42154 & 18.05567 & 19.89615 & 0.01084 & 219.38300 \\
\hline 18.41720 & 18.07555 & $\ldots$ & 0.03784 & 221.36005 \\
\hline 18.46006 & 18.09469 & 19.93032 & 0.03836 & 227.41000 \\
\hline 18.45907 & 18.11377 & 19.93880 & 0.09371 & 228.37800 \\
\hline 18.40563 & 18.07210 & $\ldots$ & 0.06348 & 235.38689 \\
\hline 18.40995 & 18.08187 & $\cdots$ & 0.01825 & 239.39029 \\
\hline 18.45908 & 18.12662 & 19.93795 & 0.01000 & 247.42200 \\
\hline 18.44071 & 18.10729 & 19.90479 & 0.01006 & 254.39000 \\
\hline 18.42990 & 18.07243 & $\cdots$ & 0.09117 & 260.41507 \\
\hline 18.48749 & 18.09320 & $\ldots$ & 0.06695 & 276.40500 \\
\hline
\end{tabular}


Table A.3. Photometry of two images of SDSS J1206+4332 quasar and a reference star.

\begin{tabular}{ccccc}
\hline \hline $\begin{array}{c}\mathrm{A} \\
(\mathrm{mag})\end{array}$ & $\begin{array}{c}\mathrm{B} \\
(\mathrm{mag})\end{array}$ & $\begin{array}{c}\text { Ref } \\
(\mathrm{mag})\end{array}$ & $\begin{array}{c}\text { Error } \\
(\mathrm{mag})\end{array}$ & $\begin{array}{c}\text { JD } \\
(\mathrm{days})\end{array}$ \\
\hline 19.50958 & 19.09693 & 19.56253 & 0.06269 & 6.72319 \\
19.45822 & 19.03115 & 19.57550 & 0.07552 & 12.73698 \\
19.48067 & 19.02927 & 19.53977 & 0.04038 & 16.66442 \\
19.48107 & 19.03157 & 19.50151 & 0.01003 & 18.69594 \\
19.49867 & 19.03066 & 19.57234 & 0.07239 & 48.69698 \\
19.44595 & 18.96723 & 19.49718 & 0.01058 & 50.66706 \\
19.45269 & 18.98587 & 19.51943 & 0.02128 & 53.73665 \\
19.49059 & 19.00116 & 19.45300 & 0.04867 & 55.77248 \\
19.44979 & 19.01361 & 19.52655 & 0.02777 & 61.65876 \\
19.43871 & 19.04837 & 19.52940 & 0.03044 & 73.79091 \\
19.56354 & 19.17763 & 19.51211 & 0.01522 & 88.59046 \\
19.49095 & 19.11235 & 19.51688 & 0.01907 & 92.76935 \\
19.34573 & 19.14561 & 19.45415 & 0.04755 & 126.41778 \\
19.31898 & 19.13856 & 19.53294 & 0.03381 & 132.41905 \\
19.26519 & 19.09754 & 19.59785 & 0.09772 & 169.38858 \\
19.34632 & 19.16507 & 19.52582 & 0.02709 & 177.49348 \\
19.34139 & 19.15273 & 19.58794 & 0.08787 & 178.38291 \\
19.44408 & 19.13593 & 19.52271 & 0.02423 & 204.39591 \\
19.45670 & 19.13912 & 19.49413 & 0.01193 & 206.47791 \\
19.47649 & 19.16528 & 19.52059 & 0.02231 & 220.46142 \\
19.45824 & 19.15208 & 19.46767 & 0.03445 & 224.38148 \\
19.46300 & 19.13344 & 19.52840 & 0.02950 & 226.41176 \\
19.48913 & 19.15389 & 19.54483 & 0.04531 & 230.41777 \\
19.49954 & 19.15120 & 19.37749 & 0.12355 & 232.41145 \\
19.47514 & 19.12036 & 19.34195 & 0.15900 & 234.46066 \\
19.49076 & 19.10947 & 19.53410 & 0.03492 & 240.41984 \\
19.50608 & 19.09624 & 19.49860 & 0.01020 & 244.40898 \\
19.50010 & 19.02842 & 19.39023 & 0.11086 & 265.40616 \\
19.44934 & 18.98559 & 19.51225 & 0.01532 & 269.39189 \\
19.50272 & 19.03939 & 19.47398 & 0.02847 & 281.37866 \\
19.50741 & 19.05486 & 19.30799 & 0.19291 & 283.37888 \\
\hline & & & & \\
& & &
\end{tabular}

Table A.4. Photometry of two images of SDSS J1335+1138 quasar and a reference star.

\begin{tabular}{ccccc}
\hline \hline $\begin{array}{c}\text { A } \\
(\mathrm{mag})\end{array}$ & $\begin{array}{c}\mathrm{B} \\
(\mathrm{mag})\end{array}$ & $\begin{array}{c}\text { Ref } \\
(\mathrm{mag})\end{array}$ & $\begin{array}{c}\text { Error } \\
(\mathrm{mag})\end{array}$ & $\begin{array}{c}\text { JD } \\
\text { (days) }\end{array}$ \\
\hline 18.10303 & 19.36676 & $\ldots$ & 0.02000 & 30.724810 \\
18.17384 & 19.44561 & $\ldots$ & 0.02000 & 35.773720 \\
18.16804 & 19.50764 & 19.81195 & 0.01724 & 39.739070 \\
18.13833 & 19.48029 & 19.82797 & 0.01019 & 42.774090 \\
18.15813 & 19.43432 & $\ldots$ & 0.02000 & 43.732740 \\
18.11639 & 19.33848 & $\ldots$ & 0.02000 & 49.774310 \\
18.12854 & 19.50847 & 19.83576 & 0.01397 & 55.622420 \\
18.13299 & 19.46182 & $\ldots$ & 0.02000 & 61.534550 \\
18.09453 & 19.43073 & $\ldots$ & 0.02000 & 67.730140 \\
18.10978 & 19.46115 & 19.81666 & 0.01368 & 100.67228 \\
18.10581 & 19.43912 & $\ldots$ & 0.02000 & 106.51100 \\
18.10918 & 19.38193 & $\ldots$ & 0.02000 & 110.62587 \\
18.09820 & 19.39721 & $\ldots$ & 0.02000 & 114.58660 \\
18.11080 & 19.39830 & $\ldots$ & 0.02000 & 116.54903 \\
18.13728 & 19.43501 & $\ldots$ & 0.02000 & 120.53586 \\
18.09940 & 19.50106 & 19.81047 & 0.01847 & 126.52229 \\
18.10319 & 19.39723 & $\ldots$ & 0.02000 & 134.55204 \\
18.08687 & 19.40392 & $\ldots$ & 0.02000 & 136.39267 \\
18.10141 & 19.44089 & $\ldots$ & 0.02000 & 138.40052 \\
18.09454 & 19.43453 & $\ldots$ & 0.02000 & 140.38453 \\
18.09550 & 19.47376 & 19.82822 & 0.01024 & 155.41550 \\
18.10153 & 19.47555 & 19.83505 & 0.01348 & 157.45223 \\
18.07648 & 19.35253 & $\ldots$ & 0.02000 & 164.41317 \\
18.08241 & 19.44983 & 19.82496 & 0.01003 & 179.41976 \\
18.08667 & 19.44891 & 19.82685 & 0.01797 & 181.40853 \\
18.08846 & 19.44335 & 19.84094 & 0.01127 & 183.41002 \\
18.06329 & 19.42084 & 19.82081 & 0.01580 & 199.42655 \\
18.01744 & 19.39086 & 19.83822 & 0.08079 & 213.41659 \\
18.05249 & 19.41707 & 19.74583 & 0.01202 & 214.39304 \\
18.04536 & 19.37535 & 19.83268 & 0.02091 & 228.41725 \\
18.06943 & 19.32710 & 19.84438 & 0.01603 & 232.38603 \\
18.05761 & 19.39882 & 19.83854 & 0.03812 & 234.37637 \\
\hline & & & & \\
& & $\ldots$ & $\ldots$
\end{tabular}

Table A.5. Photometry of two images of SDSS J1353+0118 quasar and a reference star.

\begin{tabular}{|c|c|c|c|c|}
\hline $\begin{array}{c}\mathrm{A} \\
(\mathrm{mag})\end{array}$ & $\begin{array}{c}\mathrm{B} \\
(\mathrm{mag})\end{array}$ & $\begin{array}{c}\text { Ref } \\
(\mathrm{mag})\end{array}$ & $\begin{array}{l}\text { Error } \\
\text { (mag) }\end{array}$ & $\begin{array}{c}\text { JD } \\
\text { (days) }\end{array}$ \\
\hline 17.05814 & 18.07872 & $\cdots$ & 0.06275 & 35.76942 \\
\hline 17.02775 & 18.10514 & 18.37445 & 0.03597 & 39.74420 \\
\hline 17.00462 & 18.07799 & 18.42824 & 0.02167 & 42.77780 \\
\hline 16.96852 & 18.02390 & $\cdots$ & 0.06911 & 43.72839 \\
\hline 17.03687 & 18.07236 & $\cdots$ & 0.05862 & 49.77080 \\
\hline 17.03669 & 18.07700 & 18.42902 & 0.02237 & 53.68090 \\
\hline 17.01645 & 18.08524 & 18.39505 & 0.01716 & 55.62640 \\
\hline 16.99037 & 18.02568 & $\cdots$ & 0.05730 & 59.57378 \\
\hline 17.05865 & 18.08877 & $\cdots$ & 0.04800 & 61.52997 \\
\hline 16.96488 & 18.01724 & $\cdots$ & 0.04040 & 67.73733 \\
\hline 16.98115 & 18.01752 & 18.41577 & 0.01207 & 100.67650 \\
\hline 17.10905 & 18.19464 & $\ldots$ & 0.16417 & 106.51640 \\
\hline 16.99332 & 18.07777 & $\cdots$ & 0.02836 & 110.62981 \\
\hline 17.00271 & 18.10740 & $\cdots$ & 0.02494 & 114.59100 \\
\hline 17.02668 & 18.11799 & $\cdots$ & 0.05477 & 116.55300 \\
\hline 17.00286 & 18.08704 & $\cdots$ & 0.02309 & 120.53156 \\
\hline 16.97671 & 18.02995 & $\cdots$ & 0.02077 & 122.53589 \\
\hline 16.97076 & 18.15163 & 18.41465 & 0.01148 & 126.52910 \\
\hline 16.99034 & 18.08779 & $\cdots$ & 0.02733 & 140.38854 \\
\hline 16.96808 & 18.05136 & 18.33605 & 0.07363 & 155.42000 \\
\hline 16.97024 & 18.02696 & 18.38489 & 0.02610 & 173.44620 \\
\hline 16.96620 & 18.06321 & 18.34458 & 0.06519 & 175.39800 \\
\hline 16.95872 & 18.02472 & 18.40082 & 0.01291 & 179.41520 \\
\hline 16.97581 & 18.05070 & 18.38525 & 0.02577 & 181.40410 \\
\hline 16.97564 & 18.03723 & 18.36668 & 0.04348 & 183.40570 \\
\hline 17.00969 & 18.10988 & $\cdots$ & 0.02826 & 185.39787 \\
\hline 16.97429 & 18.01590 & 18.40489 & 0.01081 & 189.42540 \\
\hline 16.95353 & 18.01173 & 18.43362 & 0.02657 & 199.43070 \\
\hline 16.92171 & 18.00251 & $\cdots$ & 0.09920 & 201.43826 \\
\hline 16.95048 & 17.99446 & 18.49120 & 0.08280 & 213.42560 \\
\hline 16.96991 & 18.04170 & 18.44207 & 0.03454 & 214.38850 \\
\hline 16.96352 & 18.03753 & 18.44432 & 0.03668 & 216.42800 \\
\hline 16.97691 & 18.05624 & 18.38822 & 0.02306 & 220.41840 \\
\hline 16.94612 & 18.03519 & 18.39483 & 0.01734 & 228.42160 \\
\hline 16.96077 & 18.07000 & 18.41878 & 0.01398 & 232.38040 \\
\hline 16.96152 & 18.02551 & 18.41092 & 0.01018 & 234.37150 \\
\hline 16.95068 & 18.01591 & 18.42423 & 0.01821 & 238.35420 \\
\hline 16.95316 & 18.03111 & $\cdots$ & 0.07028 & 240.37300 \\
\hline
\end{tabular}

\section{References}

Burud, I., Hjorth, J., Jaunsen, A. O., et al. 2000, ApJ, 544, 117 Burud, I., Courbin, F., Magain, P., et al. 2002a, A\&A, 383, 71 Burud, I., Hjorth, J., Courbin, F., et al. 2002b, A\&A, 391, 481 Chang, K., \& Refsdal, S. 1979, Nature, 282, 561 Coles, J. 2008, ApJ, 679, 17

Dalal, N., \& Kochanek, C. S. 2002, ApJ, 572, 25

Eigenbrod, A., Courbin, F., Dye, S., et al. 2006a, A\&A, 451, 747

Eigenbrod, A., Courbin, F., Meylan, G., et al. 2006b, A\&A, 451, 759

Elíasdóttir, Á., Hjorth, J., Toft, S., Burud, I., \& Paraficz, D. 2006, ApJ, 166, 443 Falco, E., Gorenstein, M. V., \& Shapiro, I. I. 1985, ApJ, 289, L1

Faure, C., Courbin, F., Kneib, J. P., et al. 2002, A\&A, 386, 69

Fohlmeister, J., Kochanek, C. S., Falco, E. E., et al. 2008, ApJ, 676, 761

Freedman, W. L., Madore, B. F., \& Gibson, B. K. 2001, ApJ, 553, 47

Golse, G., \& Kneib, J. P. 2002, A\&A, 390, 821

Hjorth, J., Burud, I., Jausen, A. O., et al. 2002, ApJ, 572, 11

Inada, N., Oguri, M., Becker, R. H., et al. 2006, AJ, 131, 1934

Jakobsson, P., Hjorth, J., Burud, I., et al. 2005, A\&A, 431, 103

Johnston, D. E., Richards, G. T., Frieman, J. A., et al. 2003, ApJ, 131, 1934

Jullo, E., Kneib, J. P., Limousin, M., et al. 2007, NJPh, 9, 447

Keeton, C. R. 2001, ApJ, submitted [arXiv: astro-ph/0102340]

Keeton, C. R., Kochanek, C. S., \& Falco, E. E. 1998, ApJ, 509, 561

Kochanek, C. S. 1993, ApJ, 419, 12

Koopmans, L. V. E., de Bruyn, A. G., Xanthopoulos, E., et al. 2000, A\&A, 356, 391

Koopmans, L. V. E., Treu, T., Bolton, A. S., et al. 2006, ApJ, 649, 599

Kormann, R., Schneider, P., \& Bartelmann, M. 1994, A\&A, 284, 285 
Magain, P., Courbin, F., \& Sohy, S. 1998, ApJ, 494, 472 Oguri, M. 2007, ApJ, 660, 1

Oguri, M., Inada, N., Castander, F. J., et al. 2004, PASJ, 56, 399

Oguri, M., Inada, N., Hennawi, J. F., et al. 2005, ApJ, 622, 106 Paczyński, B. 1986, ApJ, 301, 503

Paraficz, D., Hjorth, J., Burud, I., et al. 2006, A\&A, 455, 1

Refsdal, S. 1964, MNRAS, 128, 307

Romanowsky, A. J., \& Kochanek, C. S. 1999, ApJ, 516, 18

Rusin, D., Kochanek, C. S., \& Keeton, C. R. 2003, ApJ, 595, 29

Saha, P., \& Williams, L. L. R. 1997, MNRAS, 292, 148
Saha, P., \& Williams, L. L. R. 2004, AJ, 127, 2604

Saha, P., Coles, J., Macció, A. V., et al. 2006, ApJ, 650, 17

Schild, R. 1996, AAS, 189, 2702

Webb, W., \& Malkan, M. 2000, ApJ, 540, 652

Wold, M., Brotherton, M. S., \& Shang, Z. 2007, MNRAS, 375, 989

Wucknitz, O., \& Refsdal, S. 2001, ASPC, 237, 157

Wyithe, J. S. B., \& Turner, E. L. 2001, MNRAS, 320, 21

de Vries, W. H., Becker, R. H., \& White, R. L. 2003, AJ, 126, 1217

de Vries, W. H., Becker, R. H., \& White, R. L. 2006, ASPC, 360, 29

Vuissoz, C., Courbin, F., Sluse D., et al. 2007, A\&A, 464, 845 